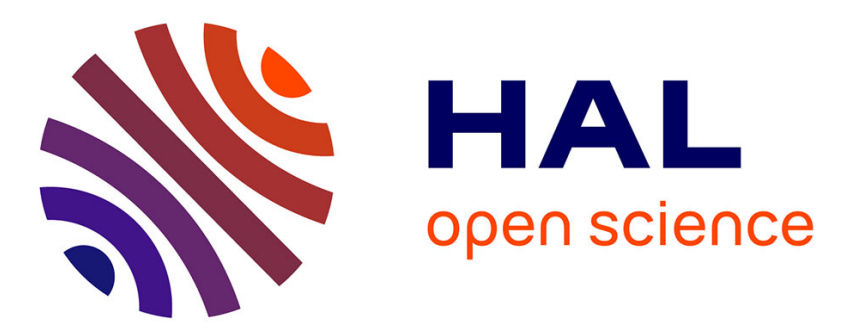

\title{
How Solutes Modify the Thermodynamics and Dynamics of Filling and Emptying in Extreme Ink-Bottle Pores
}

Olivier O. Vincent, Jiamin Zhang, Eugene Choi, Siyu Zhu, Abraham Stroock

\section{To cite this version:}

Olivier O. Vincent, Jiamin Zhang, Eugene Choi, Siyu Zhu, Abraham Stroock. How Solutes Modify the Thermodynamics and Dynamics of Filling and Emptying in Extreme Ink-Bottle Pores. Langmuir, 2019, 35 (8), pp.2934-2947. 10.1021/acs.langmuir.8b03494 . hal-02364969

\section{HAL Id: hal-02364969 \\ https://univ-lyon1.hal.science/hal-02364969}

Submitted on 10 May 2020

HAL is a multi-disciplinary open access archive for the deposit and dissemination of scientific research documents, whether they are published or not. The documents may come from teaching and research institutions in France or abroad, or from public or private research centers.
L'archive ouverte pluridisciplinaire HAL, est destinée au dépôt et à la diffusion de documents scientifiques de niveau recherche, publiés ou non, émanant des établissements d'enseignement et de recherche français ou étrangers, des laboratoires publics ou privés. 


\title{
How solutes modify the thermodynamics and dynamics of filling and emptying in extreme
} ink-bottle pores

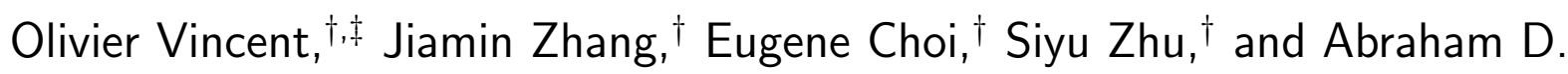 \\ Stroock $k^{*, \dagger}$ \\ $\dagger$ Robert Frederick Smith School of Chemical and Biomolecular Engineering, Cornell \\ University, 120 Olin Hall, Ithaca NY 14853 \\ $\ddagger$ Present address: Univ Lyon, Université Claude Bernard Lyon 1, CNRS, Institut Lumière \\ Matière, F-69622, Villeurbanne, France \\ E-mail: ads10@cornell.edu
}

\begin{abstract}
We investigate the filling and emptying of extreme ink-bottle porous media micrometer-scale pores connected by nanometer-scale pores - when changing the pressure of the external vapor, in a case where the pore liquid contains solutes. These phenomena are relevant in diverse contexts, such as the weathering of building materials and artwork, aerosol formation in the atmosphere, and the hydration of soils and plants. Using model systems made of vein-shaped microcavities interconnected by a mesoporous matrix, we show experimentally that the presence of nonvolatile solute shifts the condensation and evaporation transitions and in a way that is consistent with a modified Kelvin-Laplace equation that takes into account the osmotic pressure of the solution. Emptying occurs far below saturation, when the Kelvin stress, mediated by the large curvature of the liquid-vapor interfaces in the nanopores, is large enough to
\end{abstract}


induce spontaneous bubble nucleation in the micro-veins. Filling, on the other hand, occurs close to equilibrium (i.e., at saturation, $p_{\text {sat }}$ for pure water and $p_{\mathrm{s}}<p_{\text {sat }}$ for a solution), driven by the weak capillary pressure of the liquid-vapor interface in the micro-veins. Interestingly, solutes allow the system to reach situations where the vapor is supersaturated with respect to the solution $\left(p_{\mathrm{s}}<p<p_{\mathrm{sat}}\right)$. We show that in that latter situation, a condensation layer covers the outer surface of the porous system, preventing the generation of Kelvin stresses but inducing osmotic stresses and flows that are vapor-pressure-dependent. The timescales and dynamics reflect these different driving forces: emptying proceeds through discrete, stochastic nucleation events with very fast, unsteady bubble growth associated with a poroelastic relaxation process, while filling occurs collectively in all veins of the sample through a slower steady-state process driven by a combination of osmosis and capillarity. The dynamics can however be rendered symmetrical between filling and emptying if bubbles pre-exist during emptying, a case that we explore using cycling of the vapor pressure around equilibrium.

\section{Introduction}

Porous media are ubiquitous in nature and technology, and the way they uptake and transport water plays crucial roles in many contexts, such as the stability of food products, ${ }^{1}$ formation of clouds, ${ }^{2}$ transport of sap in plants, ${ }^{3}$ mechanics of granular materials, ${ }^{4}$ or the fabrication and aging of concrete. ${ }^{5}$ In many situations, the pore fluid contains dissolved species, and the presence of these solutes is thought to have specific effects, such as osmosisdriven refilling of embolized vessels in plants ${ }^{6}$ or damage in rocks or building materials due to osmotic stress ${ }^{7}$ or crystallization-induced stresses. ${ }^{8}$ The thermodynamics and kinetics of hydration in porous media is still an area of active research, with open questions about the validity of continuum laws at small scales ${ }^{9,10}$ or the role of pore architecture and disorder. ${ }^{11,12}$

Experimental efforts to answer these questions have relied on the fabrication of model

porous strucures with well-defined homogeneous pore shape and sizes, ${ }^{12,13}$ or with controlled 
disorder, such as ink-bottle structures that exhibit large cavities connected by narrower pores ${ }^{11,14,15}$ (Figure 1a). Ink-bottle structures are associated with the generation of strongly metastable states (large, negative pressures) of the pore liquid during desorption, leading to relaxation by bubble nucleation (cavitation) in the larger pores (Figure 1a). ${ }^{11,15}$ Recently, we proposed a new type of extreme ink-bottle porous medium, where several orders of magnitude separate pore size $\left(r_{\mathrm{p}} \sim \mathrm{nm}\right)$ and cavitiy size $\left(H_{\mathrm{v}} \sim \mu \mathrm{m}\right) .{ }^{16}$ Such a structure mimics the architecture of vascular systems in plants ${ }^{3}$ and is more generally a model of highly heterogeneous porous media such as rocks, ${ }^{17}$ soils, ${ }^{18}$ or concrete. ${ }^{7}$ With such extreme-ink bottle systems, we showed that we could directly visualize the dynamics of of condensation and evaporation, including cavitation, owing to direct optical access into the larger cavities. ${ }^{16}$

A large number of existing theories for adsorption and desorption in pores are based on Kelvin-Laplace equation, ${ }^{9,19,20}$ which relates the capillary stress (liquid pressure, $P<0$, Figure 1a) in the pore fluid to the sub-saturation of the vapor in contact with the porous medium $\left(p / p_{\text {sat }}<1\right)$. One may wonder how the presence of solutes in the pore liquid (Figure 1a) affects these thermodynamic phenomena - phase equilibrium and capillarity - and the transient filling and emptying dynamics in the porous medium, with specific questions about the relative effect of the stress implied by Kelvin-Laplace equation and that of osmotic pressure, or the effects of pore architecture and solute identity. ${ }^{21,22}$

Here, we report on static and dynamic experiment with extreme ink-bottle porous media (microscale veins interconnected by nanopores) in the presence of solutes. We focus on the liquid/vapor phase transition in the microscale cavities (veins), induced by changes in external water vapor pressure, in a regime where the nanopores stay filled with liquid (no desorption in the nanopores). To avoid confusion with desorption and condensation in the nanoporous layer itself (which will be addressed in a future paper), we will describe the behavior of the micro-veins as filling and emptying.

After describing our experimental methods, we develop a theoretical basis for predicting the thermodynamics and dynamics of ink-bottle pores filled with solute and subject to vapor 
pressure changes. In the Theory section, we first adapt classical thermodynamical treatments of phase equilibrium to account for the combined impacts of large capillary stresses (KelvinLaplace) and solution (osmotic equilibrium). We then turn to treatments of the dynamics: first we consider drying of the material by cavitation of the solution in the micro-veins and subsequent emptying driven by large tensile stresses and poroelastic relaxation; second, we consider filling and emptying by osmotic processes in cases when a film of solution forms on the external surface. We finally present and discuss our experimental observations for the thermodynamics (isotherms and evaporation/condensation hysteresis) and dynamics (filling and emptying flows) in extreme ink-bottle structures, in relation to the theoretical elements established previously.

\section{Materials and methods}

We created the extreme ink-bottle structures (Figure 1b-c) from silicon wafers (p-type, 1-10 S.cm, $\langle 111\rangle$ crystal orientation) and borosilicate glass wafers. In the silicon, we etched channels ( $W=10 \mu \mathrm{m}$-wide, $H_{\mathrm{v}}=3 \mu \mathrm{m}$-deep, $L=2 \mathrm{~mm}$-long, see Figure $1 \mathrm{~b}-\mathrm{c}$ ) using photolithography and dry, plasma-etching. We then proceeded to porosify a uniform layer of thickness, $H_{\mathrm{p}}=15 \mu \mathrm{m}$, in the side of the silicon wafer presenting these channels, using anodization in a 1:149\% $\mathrm{HF}$ : ethanol electrolyte at current density $20 \mathrm{~mA} / \mathrm{cm}^{2}$. Anodization resulted in the formation of an array of laterally connected mesopores $\sim 3 \mathrm{~nm}$ in diameter and of porosity $\phi=0.45$, as estimated from nitrogen porosimetry and water imbibition and permation measurements. ${ }^{23}$ We bonded the porosified silicon surface to glass to form the extreme ink-bottle structure, i.e. microchannels (referred as veins below) indirectly connected both to each other and to the outside through nanopores (Figure 1b-c). Note that since the etch depth of the nanoporous layer $\left(H_{\mathrm{p}}=15 \mu \mathrm{m}\right)$ was greater than the depth of the veins $\left(H_{\mathrm{v}}=3 \mu \mathrm{m}\right)$, the veins were surrounded by the nanoporous matrix in the three directions of space, with non-porous glass defining the top boundary. 
(a) ideal ink-bottle situation

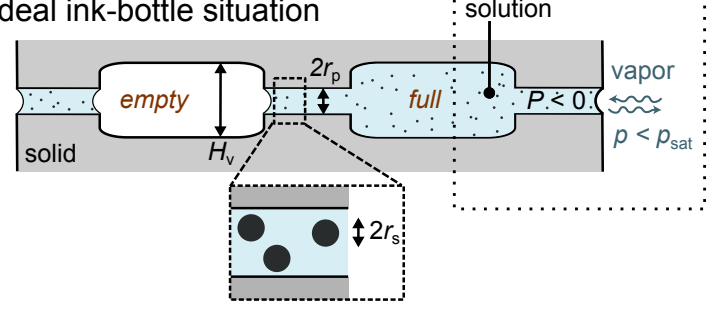

(b) actual sample (cross section sketch)

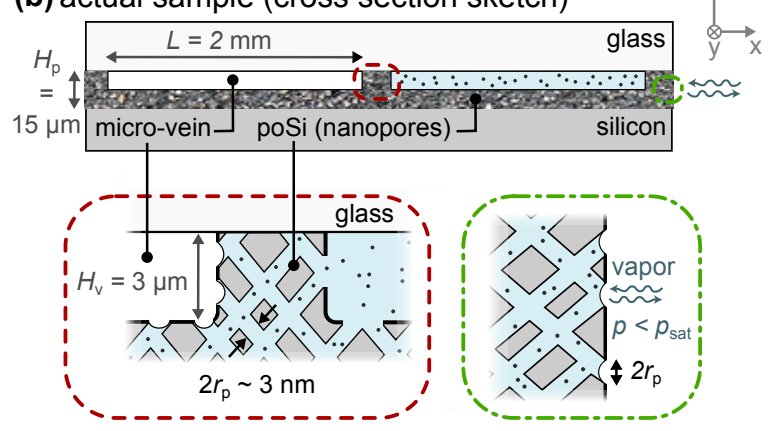

(c) actual sample (top-view photograph)

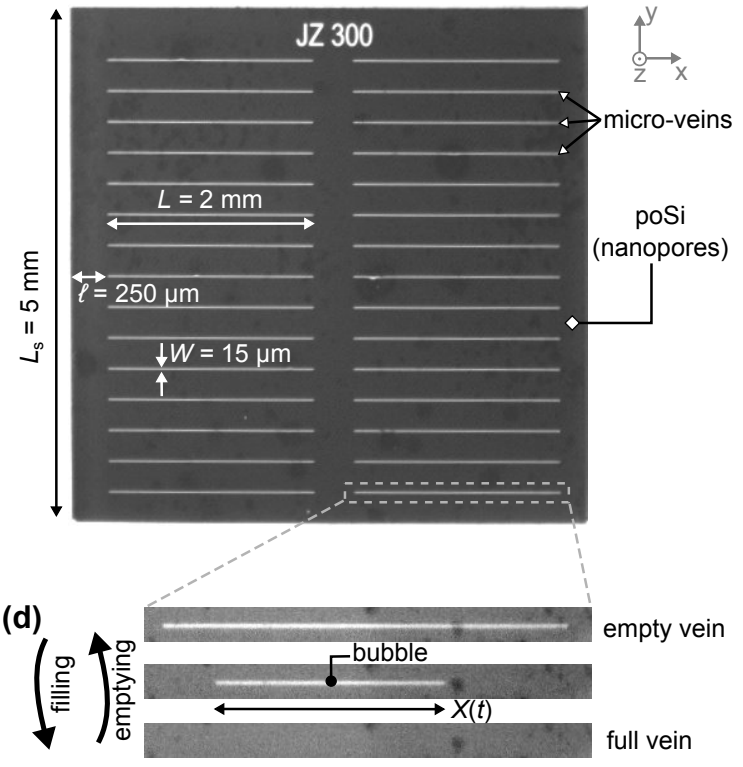

Figure 1: Experimental system. (a) Generic sketch of an ink-bottle geometry with large voids (width, $H_{\mathrm{v}}$ ) connected by small pores (pore radius, $r_{\mathrm{p}}$ ). The solid and liquid are sketched in gray and light blue, respectively. In this paper we consider a case where the structure is filled with a solution (solute is represented as black dots, of hydrodynamic radius, $r_{\mathrm{s}}$ ) and where only the voids fill and empty (no desorption in the nanopores). Hypothetical phase behavior and dynamics in such a situation are represented in Figure 2. The zone delimited by the dotted line is the one depicted in Figure 2. (b) Cross-section sketch of our actual samples, with extreme ink-bottle geometry: the voids are vein-shaped with dimensions in the micrometer range $\left(H_{\mathrm{v}}=3 \mu \mathrm{m}\right)$, while the porous layer (porous silicon, poSi) interconnecting the voids consists in a network of pores in the nanometer range (diameter, $2 r_{\mathrm{p}} \sim 3 \mathrm{~nm}$ ). Insets represent the nanoporous area (not to scale) between veins (red dashes) and at the sample edge (green dash-dots). Samples are placed in an environment with controlled, subsaturated vapor pressure, $p<p_{\text {sat. }}$. (c) Top-view photograph of a sample. (d) The vein-shaped microcavities allow for optical tracking of filling and emptying. 
We filled the sample by immersing it in liquid for at least two days. For the liquid, we used pure water and aqueous solutions of both urea and lithium chloride. The main results described in the present paper were obtained with a urea solution of activity $p_{\mathrm{s}} / p_{\text {sat }}=$ $0.828 \pm 0.004$ (osmotic pressure $\Pi_{\text {in }}=25.0 \pm 0.7 \mathrm{MPa}$ ), as measured in situ (see SI, section 4). Similar results obtained using lithium chloride solutions of various concentrations are described in the SI (section 5).

We ran isothermal experiments at temperature $T=15^{\circ} \mathrm{C}$ in a pure water vapor environment of controlled vapor pressure $p$, using a thermostated vacuum chamber connected to a water vapor source. Details on this vacuum system can be found elsewhere. ${ }^{24,25} \mathrm{~A}$ glass window in the vacuum chamber allowed optical monitoring of the sample: veins filled with liquid appeared dark, while veins filled with vapor were highly reflective (Figure 1d). The filling state and the dynamics of the liquid in the veins as a function of the imposed thermodynamic conditions (vapor pressure) were obtained by image analysis by tracking the position of the menisci in image sequences as in Figure 1d.

The geometry of the sample was such that the total volume of veins $\left(V_{\mathrm{v}}=N \times W \times L \times H_{\mathrm{v}}\right)$ was small compared to the total volume of the nanopores $\left(V_{\mathrm{p}}=\phi \times L_{\mathrm{s}}^{2} \times H_{\mathrm{p}}\right)$, with $N=30$ the total number of veins, and $L_{\mathrm{s}}=5 \mathrm{~mm}$ the lateral dimension of the sample. Since $V_{\mathrm{v}} / V_{\mathrm{p}} \simeq 0.01$, the solute concentration (equivalently, the osmotic pressure, $\Pi_{\text {in }}$ ) in the sample depended only weakly on the filling state of the veins, and we will assume $\Pi_{\text {in }}$ constant in the following.

For simplicity, we will use ideal sketches as in Figure 1a to discuss the physical processes at play in our experiments, i.e., with a single nanopore connecting a micro-vein to the outside. This sketch of a single nanopore represents, in reality, a large area of nanoporous matrix situated between the micro-vein and the sample edge (across the distance $\ell$ in Figure 1c), the porous layer contains a large density of nanopores that form an interconnected, isotropic pore space. The typical cross-section of nanoporous material connecting each vein to the outside is $\mathcal{A} \sim d \times H_{\mathrm{p}}$, where $d=300 \mu \mathrm{m}$ is the separation between veins in the 
$y$ direction, as defined in Figure 1b-c. A typical number of pores in that cross section is $N_{\mathrm{p}} \sim \mathcal{A} \phi /\left(\pi r_{\mathrm{p}}^{2}\right) \sim 10^{8}$ so that every micro-vein in our sample is connected to the outside (and to the neighboring micro-veins) through a very large number of nanopores, allowing us to use macroscopic, averaged transport coefficients (e.g. the Darcy permeability).

From the value of the Darcy permeability, $\kappa\left[\mathrm{m}^{2} /(\mathrm{Pa} . \mathrm{s})\right]$, measured in similar samples with pure water, ${ }^{23,25}$ we estimate the intrinsic permeability $k=\eta_{\mathrm{w}} \kappa=(1.77 \pm 0.12) \times 10^{-20} \mathrm{~m}^{2}$ of the nanoporous layer, where $\eta_{\mathrm{w}}$ (Pa.s) is the viscosity of water. From literature values, we estimated the ratio of viscosities $\eta_{\mathrm{s}} / \eta_{\mathrm{w}}=1.54$ between the urea solution and pure liquid water. ${ }^{26}$ We also estimated the hydraulic radius of the urea molecule, $r_{\mathrm{s}}=0.17 \mathrm{~nm}$, from its diffusivity in water ${ }^{27}$ and the Stokes-Einstein relation.

\section{Theory}

Here, we describe the theoretical framework for our study, using sketches such as in Figure 1a, focusing on the area inside the dotted rectangle. All sketches of the physical situations considered in this paper are in Figure 2. As discussed in the Methods section, the sketched nanopore actually represents a large number of nanopores in our experimental samples, of total volume much larger than the micro-vein itself. We recall that an important consequence of this experimental geometry is that filling-emptying processes in the micro-veins do not change appreciably the solute concentration inside the sample, such that the internal osmotic pressure, $\Pi_{\text {in }}$, is constant.

First, we derive a modified Kelvin equation that describes the metastable equilibrium between a solution and subsaturated vapor. Second, we briefly describe the bubble nucleation (cavitation) process that occurs as a relaxation of this metastable state. Last, we establish the fluxes in response to hydrostatic and osmotic pressures in dynamic situations. We use continuum theories of thermodynamics and fluid mechanics, and we assume that the fluid behaves as a bulk fluid, even in the nanopores. We have shown previously that this 
assumption holds for pure liquids in this type of nanopores. ${ }^{25}$ We also neglect limitations to mass transfer in the vapor phase; this assumption is justified by the geometry and low permeability of the porous layer, such that transport limitations are within the sample itself and not in an external boundary layer, ${ }^{23}$ and by the use of a vacuum system to eliminate diffusion-limited transport in the vapor phase. ${ }^{25}$

\section{Thermodynamics of solution-vapor equilibrium}

We consider a situation as sketched in Figure 2a, where the solution filling the pore (pressure, $P[\mathrm{~Pa}])$ is in equilibrium with the pure vapor of the solvent (pressure, $p[\mathrm{~Pa}]$ ) across a meniscus of radius of curvature, $r[\mathrm{~m}]$. The solution is characterized by its osmotic pressure $\Pi(C)[\mathrm{Pa}]$, or its equilibrium bulk vapor pressure $p_{\mathrm{s}}(C)[\mathrm{Pa}]$, which both depend on the concentration $C\left[\mathrm{~mol} / \mathrm{m}^{3}\right]$ of solute. We consider that the solutes of interest are non-volatile. The condition of isothermal equilibrium between the pore solution and the vapor is the equality of the chemical potential $\mu_{\mathrm{w}}[\mathrm{J} / \mathrm{mol}]$ of the solvent in the solution and in the vapor phase. The equality of pressure does not hold, in general, across the pore-confined liquidvapor interface, due to capillary effects $(P \neq p$ in general). We consider isothermal processes at temperature, $T[\mathrm{~K}]$.

Liquid-vapor equilibrium We first recall the expressions of the chemical potential of pure solvent, in the vapor state (vapor pressure, $p[\mathrm{~Pa}]$, chemical potential, $\mu_{\mathrm{w}}^{\mathrm{vap}}[\mathrm{J} / \mathrm{mol}]$ ) and in the liquid state (pressure, $P$, chemical potential, $\mu_{\mathrm{w}}^{\text {liq }}$ ). Equilibrium between the bulk, pure liquid and its vapor (saturation) occurs when $P=p=p_{\text {sat }}(T)$, where $p_{\text {sat }}$ is the saturation vapor pressure. We use this situation as the reference thermodynamic state of the system, with an associated reference chemical potential, $\mu_{0}(T)$. Then, the chemical potentials can be expressed, in general, as

$$
\mu_{\mathrm{w}}^{\mathrm{vap}}(p)=\mu_{0}+R T \ln \left(\frac{p}{p_{\mathrm{sat}}}\right)
$$


for the vapor, where $R T[\mathrm{~J} / \mathrm{mol}]$ is the thermal energy, and

$$
\mu_{\mathrm{w}}^{\operatorname{liq}}(P)=\mu_{0}+v_{\mathrm{w}}^{\operatorname{liq}}\left(P-p_{\mathrm{sat}}\right)
$$

for the liquid, where $v_{\mathrm{w}}^{\text {liq }}\left[\mathrm{m}^{3} / \mathrm{mol}\right]$ is the molar volume of the pure solvent. Equation 1 is obtained by assuming that the vapor behaves as an ideal gas (with molar volume $R T / p$ ). Equation 2 comes from the assumption of an incompressible liquid ( $v_{\mathrm{w}}^{\text {liq }}$ is constant, independent of $P$ ), which results in negligible errors even for pressure differences in the $10-100$ MPa range. ${ }^{23}$

The well-known Kelvin equation is obtained from the equality of chemical potentials in Equations 1 and 2:

$$
P=p_{\text {sat }}+\Psi(p)
$$

where we have defined the water potential of the vapor at pressure $p$.

$$
\Psi(p)=\frac{R T}{v_{\mathrm{w}}^{\operatorname{liq}}} \ln \left(\frac{p}{p_{\mathrm{sat}}}\right) .
$$

Physically, water potential $\Psi=\left(\mu_{\mathrm{w}}-\mu_{0}\right) / v_{\mathrm{w}}^{\text {liq }}[\mathrm{Pa}]$ is the deviation in pressure away from $p_{\text {sat }}$ for a pure liquid in metastable equilibrium with a subsaturated vapor phase. Equations 3-4 describe the necessary relation between vapor pressure $p$ and liquid pressure $P$ (or $\Psi$ ) for isothermal equilibrium between a pure liquid and its vapor.

Solution-vapor equilibrium Adding a nonvolatile solute to the liquid to form a solution (concentration, $C\left[\mathrm{~mol} / \mathrm{m}^{3}\right]$ ) modifies the equilibrium conditions established above, because the mixing of the solvent (water in our experiments) and the solute modifies the chemical potential of the solvent, while the chemical potential of the pure vapor as expressed in Equation 1 remains unchanged. One consequence of this shifted equilibrium is that the equilibrium vapor pressure, $p_{\mathrm{s}}$, of the bulk solution is different from $p_{\text {sat }}$ and depends on the concentration, $C$, of solute, with $p_{\mathrm{s}}(C)<p_{\text {sat }}$. The parameter $p_{\mathrm{s}}$ is thus a measure of the 


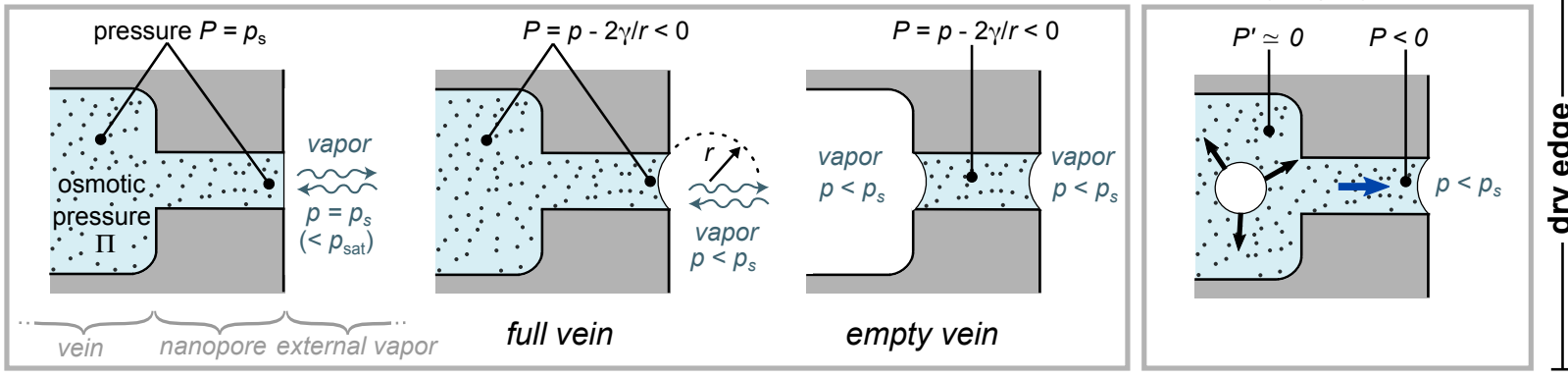

(c) Osmotic-capillary flow transients

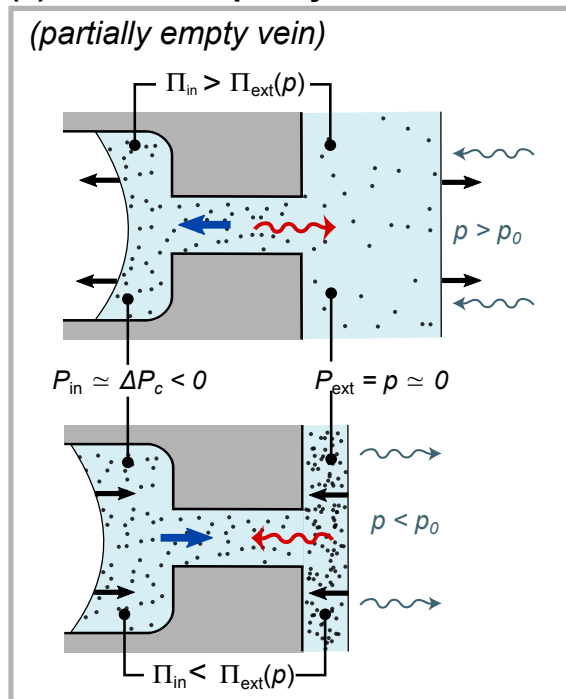

(d) Osmotic stress transients

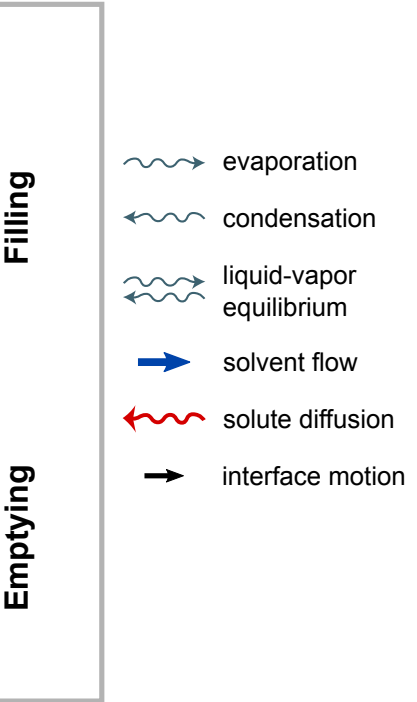

(full vein)

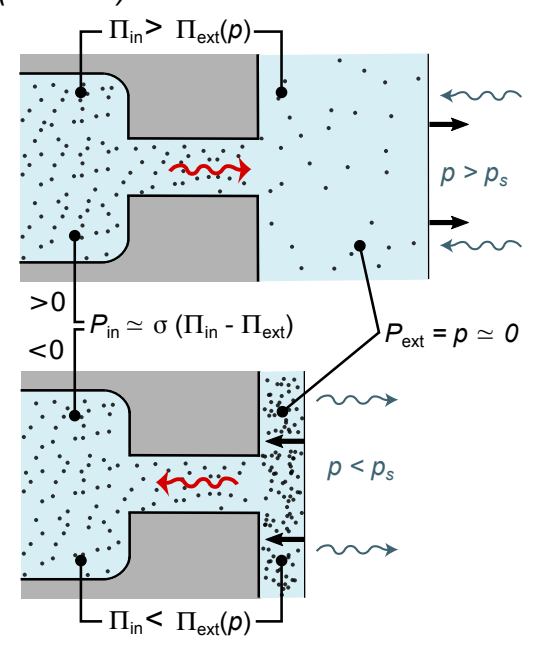

Figure 2: Theoretical sketches (not to scale) of the processes involved during filling and emptying of ink-bottle pores focusing on the area within the dotted rectangle in Figure 1a. The single nanopore sketched here for simplicity actually represents a large number of pores connecting the micro-vein to the outside (see Methods and Figure 1b), of total volume much larger than the micro-veins, so that filling and emptying of the veins does not induce appreciable concentration changes in the sample. (a) Phase equilibria governed by Kelvin equation (Equation 11): bulk equilibrium (left), metastable, negative-pressure equilibrium (center), empty vein (right). (b) Cavitation of solution in the vein due to negative-pressure metastability (as in (a), center). Cavitation leads to emptying of the vein (as in (a), right), which is governed by a fast poroelastic process (Equation 13). (c) Filling (top, for $p>p_{0}$ ) and emptying (bottom, for $p<p_{0}$ ) flows due to osmosis in partially empty veins. Due to the small effect of capillarity, $p_{0}$ is slightly shifted from $p_{\mathrm{s}}$ (Equation 22). (d) Fullvein transient situations with positive (top, for $p>p_{\mathrm{s}}$ ) and negative (bottom, for $p<p_{\mathrm{s}}$ ) stresses induced by osmosis (Equation 23). Panels (a-b) correspond to situations where the sample edge is dry, while panels (c-d) correspond to transient situations with bulk solution wetting the surface of the sample. The four panels correspond to the four sections in the Theory part of the article. 
impact of the solute on the solvent chemical potential. In the general case, also taking into account the effect of the pressure, $P$, of the solution, the chemical potential of the solvent in the solution is (see SI, section 1)

$$
\mu_{\mathrm{w}}^{\mathrm{sol}}(P, C)=\mu_{0}+R T \ln \left(\frac{p_{\mathrm{s}}(C)}{p_{\mathrm{sat}}}\right)+v_{\mathrm{w}}^{\text {liq }}\left(P-p_{\mathrm{s}}(C)\right)
$$

where $p_{\mathrm{s}}$ is the bulk equilibrium vapor pressure of the solution, and where we have used the well-verified approximations that the solution is incompressible and that the partial molar volume of the solvent in the solution is unchanged compared to the pure liquid case $\left(v_{\mathrm{w}}^{\mathrm{sol}}=v_{\mathrm{w}}^{\text {liq }}\right.$, see SI section 2$)$. Note that Equation 5 can be rewritten in a similar manner as Equation 2, i.e. $\mu_{\mathrm{w}}^{\mathrm{sol}}=\mu_{0, \mathrm{~s}}(T, C)+v_{\mathrm{w}}^{\mathrm{liq}}\left(P-p_{\mathrm{s}}\right)$, where $\mu_{0, \mathrm{~s}}(T, C)=\mu_{0}(T)+R T \ln \left(p_{\mathrm{s}}(C) / p_{\text {sat }}\right)$ is the chemical potential corresponding to bulk equilibrium between the solution and the vapor (see also SI section 1). The difference between the pure liquid reference potential $\mu_{0}$ and the solution reference potential $\mu_{0, \mathrm{~s}}$ encompasses the effect of solute and results in extra terms due to the non-cancelling of $\mu_{0}$ and $\mu_{0, \mathrm{~s}}$ when equating the vapor and solution chemical potentials, contrary to the pure liquid case.

An equivalent expression to Equation 5 is

$$
\mu_{\mathrm{w}}^{\mathrm{sol}}(P, C)=\mu_{0}+v_{\mathrm{w}}^{\text {liq }}\left(P-\Pi(C)-p_{\mathrm{sat}}\right) .
$$

where $\Pi$ is the osmotic pressure of the solution, defined as the excess pressure of the pure solvent that would be in equilibrium with the solution through a semi-permeable membrane (see SI, section 1). The osmotic pressure, $\Pi$ is related to the equilibrium vapor pressure of the solution, $p_{\mathrm{s}}$, through

$$
\Pi(C)=-\frac{R T}{v_{\mathrm{w}}^{\operatorname{liq}}} \ln \left(\frac{p_{\mathrm{s}}(C)}{p_{\mathrm{sat}}}\right)+p_{\mathrm{s}}(C)-p_{\mathrm{sat}} .
$$

The term $p_{\mathrm{s}}-p_{\text {sat }}$ is usually negligible (except for extreme dilutions of the solute, see SI 
section 1) so that the more familiar expression $\Pi \simeq-R T \ln \left(p_{\mathrm{s}} / p_{\mathrm{sat}}\right) / v_{\mathrm{w}}^{\text {liq }}$ is an excellent approximation in most practical cases.

As a result, the equality of the solvent chemical potentials in the vapor phase (Equation 1) and in the solution (Equations 5-6) now requires

$$
P=p_{\mathrm{s}}+\frac{R T}{v_{\mathrm{w}}} \operatorname{lin}\left(\frac{p}{p_{\mathrm{s}}(C)}\right)
$$

when expressed in terms of vapor pressure, and

$$
P=p_{\text {sat }}+\Psi(p)+\Pi(C)
$$

when expressed in terms of osmotic pressure. Equations 8-9 are generalizations of the Kelvin relation (Equation 3). Note that Kelvin equation for pure solvent is a special case of Equations 8-9 in the limit of infinite dilution, for which $p_{\mathrm{s}}=p_{\text {sat }}$ and $\Pi=0$. Equation 9 indicates that the presence of the solute simply shifts the liquid pressure predicted from the standard Kelvin equation by the osmotic pressure $\Pi$. In practice, the liquid pressure predicted by Kelvin equation is much larger in magnitude $(\sim \mathrm{MPa})$ than the vapor pressure $(\sim \mathrm{kPa})$ so that to a very good approximation

$$
P \simeq \frac{R T}{v_{\mathrm{w}}^{\operatorname{liq}}} \ln \left(\frac{p}{p_{\mathrm{s}}(C)}\right) \simeq \Psi(p)+\Pi(C)
$$

The pressure mismatch between the solution $(P)$ and the vapor phase $(p)$ imposed by Equations 8-9 must be accommodated by capillarity through the curvature of the liquidvapor interface, as described by the Laplace equation $P-p=-2 \gamma / r$, where $\gamma[\mathrm{N} / \mathrm{m}]$ is the liquid-vapor interfacial tension and where we have assumed that the menisci have a hemispherical shape with a radius of curvature, $r$ (Figure 2a, center). From Equations 8-9,

$$
P-p=-\frac{2 \gamma}{r}=\frac{R T}{v_{\mathrm{w}}^{\text {liq }}} \ln \left(\frac{p}{p_{\mathrm{s}}(C)}\right)+p_{\mathrm{s}}(C)-p=\Psi(p)+\Pi(C)+p_{\mathrm{sat}}-p
$$


or, neglecting vapor pressures compared to liquid and osmotic pressures,

$$
-\frac{2 \gamma}{r} \simeq \frac{R T}{v_{\mathrm{w}}^{\operatorname{liq}}} \ln \left(\frac{p}{p_{\mathrm{s}}}\right) \simeq \Psi(p)+\Pi(C) .
$$

Equations 11-12 indicate that when the pressure of the vapor, $p$, is equal to the bulk vapor pressure of the solution, $p_{\mathrm{s}}$, the pore solution is at the same pressure as the vapor $(P=$ $\left.p=p_{\mathrm{s}}\right)$, and the solution-vapor interface is flat $(1 / r=0)$, as depicted in Figure $2 \mathrm{a}$, left. In this situation, the vapor is sub-saturated with respect to the pure fluid, but saturated with respect to the solution. When $p<p_{\mathrm{s}}$ (vapor sub-saturated with respect to the solution), from Equation 11, the pore solution must be at reduced pressure $P<p$, and the meniscus at the pore mouth takes a shape as sketched in Figure 2a (center), corresponding to $r>0$. As we explain in the next section, this reduced pressure can be responsible for evaporation by cavitation (nucleation of vapor bubbles, Figure 2b) within the large pores (veins), resulting in the state with empty veins depicted in Figure 2a, right.

We also note here that if $p>p_{\mathrm{s}}$, Equation 11 predicts that the meniscus should be curved outwards $(r<0)$ to accommodate an increased pressure in the pore fluid $(P>p)$. Such a configuration, however, is not possible for a hydrophilic material, as the solution tends to spread out on the external surface instead of forming a meniscus with $r<0$; as a result Kelvin equilibrium is only possible when $p \leq p_{\mathrm{s}}$ in a hydrophilic pore.

Thus, imposing vapor conditions $p>p_{\mathrm{s}}$ where the vapor is supersaturated with respect to the solution (while still being sub-saturated with respect to the pure fluid) results in the formation of a film of solution on the outside of the porous medium as depicted in Figures 2c-d. As we we discuss below (see section Filling-emptying by osmosis and capillarity), this situation results in stresses (both positive, $P>p$, and negative, $P<p$ ), due to osmosis instead of Kelvin equation and curved nano-menisci. 


\section{Cavitation, desorption, and poroelastic emptying}

As discussed above, imposing a vapor pressure $p<p_{\text {s }}$ results in a reduced pressure $P<p$ in the pore solution. In fact, from Equation 10, the pressure quickly becomes negative $(P<0)$ as $p$ is decreased away from $p_{\mathbf{s}}$. This negative liquid pressure (tension) implies that the liquid is metastable with respect to the nucleation of vapor bubbles (cavitation, see

Figure $2 \mathrm{~b}) .{ }^{28,29}$ The probability of nucleation increases when the pressure gets more negative (tension increases) and the nucleation rate is strongly non-linear as a function of pressure so that in practice, cavitation happens only in a narrow range of pressure around a typical value $P_{\text {cav }}$. Most studies with water in various systems have reported $P_{\text {cav }}$ to be in the range -20 to $-30 \mathrm{MPa}$, however the exact physical mechanism leading to these values is still unclear, especially when compared to classical nucleation theory (CNT) of homogeneous cavitation; CNT predicts cavitation of water at much more negative pressures. While the effect of volume on cavitation pressure is expected to be small, mechanisms such as heterogeneous nucleation on less hydrophilic solid surfaces or ubiquitous hydrophobic impurities have been invoked. ${ }^{30}$ Identification of nucleation pathways is outside the scope of the present paper. As in previous work, ${ }^{16}$ we use a modified CNT expression to fit our data, where an effective surface tension is used instead of that of the bulk fluid, without committing to a specific cavitation mechanism (see SI, section 3).

As discussed in our previous work with similar extreme ink-bottle systems, ${ }^{16}$ we also consider that cavitation is possible only in the microscale cavities (veins), while confinement in the nanopores hinders nucleation. As a result, the new equilibrium state reached after cavitation is as depicted in Figure 2a (right panel): the empty vein is filled with vapor at the same pressure as the outside vapor $\left(p<p_{\mathrm{s}}\right)$, while the nanopores are still filled with solution at negative pressure following Equations 8-9; the curvature of the liquid-vapor menisci both at the vein/nanopore and at the nanopore/outside interfaces is given by Equation 11.

Emptying of the nanopores is possible by further decreasing the external vapor pressure to induce desorption. This latter process occurs when the radius of curvature, $r$, of the liquid- 
vapor interface exceeds the maximum value allowed by the geometry and local mechanical equilibrium constraints, $r_{\text {des }}=r_{\mathrm{p}} / \cos \theta$, where $\theta$ is the receding contact angle of the solution on the solid. ${ }^{16}$ Using equation 11 , desorption occurs when the vapor pressure reaches $p_{\text {des }} \simeq$ $p_{\mathrm{s}} \exp \left[-2 \sigma \cos \theta v_{\mathrm{w}}^{\text {liq }} /\left(r_{\mathrm{p}} R T\right)\right]$. For the urea solution with $p_{\mathrm{s}}=0.83 \times p_{\text {sat }}$, using conservative values $r_{\mathrm{p}}=2 \mathrm{~nm}, \sigma=0.073 \mathrm{~N} / \mathrm{m}, \theta=25^{\circ},{ }^{23}$ we estimate that desorption occurs for $p_{\text {des }} / p_{\text {sat }}<0.5$. In the experiments reported here, we kept the vapor pressure far above $p_{\text {des }}$ so that desorption does not happen. In the following, we will thus consider that the nanopores always remain full with solution as sketched in Figure 2. We will examine desorption and condensation in the nanopores in the presence of solute in an upcoming paper.

When cavitation occurs, the tension in the liquid is locally relaxed because of the formation of a bubble (Figure 2b); the negative pressure vanishes and conditions quickly reestablish around equilibrium in the vein $\left(P \simeq p_{\mathrm{s}}\right)$. However, the nanopores surrounding the vein are still filled with solution at negative pressure: as a result, there exist a pressure gradient that drives viscous flow out of the vein into the nanopores (blue arrow in Figure $2 \mathrm{~b})$. In fact, due to the very large negative pressures, these flows also involve compressibility effects: as the pressure increases in the surrounding nanoporous layer, the volume of the fluid decreases. This capacitance effect allows for transient flow into the nanopores that occur before steady-state flow to the edge of the sample is established. The coupling between viscous flow and compressibility can be modeled by Biot's theory of poroelasticity. ${ }^{16,31}$

We present in the SI (section 6) a simple poroelastic model that reproduces the bubble growth dynamics observed after cavitation in our experiments. Given the geometrical parameters of our medium (nanoporous layer of depth $H_{\mathrm{p}}$, porosity $\phi$, and intrinsic permeability $k$, micro-veins of width $W$ and depth $H_{\mathrm{v}}$ (see Figure $1 \mathrm{~b}-\mathrm{c}$ ), the predicted bubble growth (vein emptying) time is

$$
t_{\mathrm{e}}=\frac{\pi}{\phi}\left(\frac{H_{\mathrm{v}} W}{4 H_{\mathrm{p}} P_{\text {cav }}}\right)^{2} \frac{\eta}{\chi k},
$$

where $\chi\left[\mathrm{Pa}^{-1}\right]$ and $\eta[\mathrm{Pa} . \mathrm{s}]$ are the compressibility and viscosity of the liquid. The predicted poroelastic emptying time from equation 13 is $t_{\mathrm{e}}=0.76 \mathrm{~s}$ for water and $t_{\mathrm{e}}=1.17 \mathrm{~s}$ for the 
urea solution (see SI, section 6 for details).

\section{Filling-emptying by osmosis and capillarity}

When the vein is empty as in Figure 2a (right) spontaneous refilling can occur if the vapor pressure $p$ is increased above $p_{\mathrm{s}}$. As we have discussed above (Thermodynamics of solutionvapor equilibrium), there is no possible equilibrium in this situation since condensation of the vapor into the solution at the pore mouths will result in a growing liquid film on the outside of the sample (Figure 2c, top). Condensation of the vapor into that film will dilute the solution, creating an imbalance in solute concentration (i.e., an imbalance in osmotic pressure) between the inside of the sample and the outside, resulting in an osmotic driving force. This driving force is directed towards high concentrations and thus induces a flow of solvent into the sample, filling the micro-veins. The situation can be reversed when imposing a vapor pressure $p<p_{\mathrm{s}}$ : then the film evaporates, generating high concentrations at the sample surface and outwards osmotic flow emptying the micro-veins (Figure 2c, bottom). The capillary pressure of the liquid-vapor interface inside of the vein $\left(\Delta P_{\mathrm{c}}\right.$ in Figure $\left.2 \mathrm{c}\right)$ is an additional driving force, which favors filling and resists emptying.

We now quantify the effects described qualitatively above. We consider the situation sketched in Figure 2c with a film of solution covering the outer surface of the sample. Me-

chanical equilibrium imposes that the pressure, $P_{\text {ext }}$, of the solution in that film is equal to the imposed vapor pressure, $p$. We also assume that this outer liquid film achieves local thermodynamic equilibrium with the vapor, so that its chemical potential is imposed by that of the vapor. This latter condition imposes the solute concentration in the film, and thus the local osmotic pressure $\Pi_{\text {ext }}$. Indeed, from Equations 1 and 6 we have:

$$
\Pi_{\text {ext }}(p)=p-p_{\text {sat }}-\Psi(p)
$$

using the definition of the vapor water potential $\Psi(p)$ (Equation 4 ). We assume that the 
osmotic pressure inside of the veins, $\Pi_{i n}$, is that of the solution used to fill the sample initially; $\Pi_{\text {in }}$ is thus related to $p_{\mathrm{s}}$ through Equation 7. As a result, the osmotic pressure imbalance is, from Equations 4, 14, and 7

$$
\Delta \Pi=\Pi_{\mathrm{ext}}-\Pi_{\mathrm{in}}=p-p_{\mathrm{s}}-\frac{R T}{v_{\mathrm{w}}^{\operatorname{liq}}} \ln \left(\frac{p}{p_{\mathrm{s}}}\right) \simeq-\frac{R T}{v_{\mathrm{w}}^{\operatorname{liq}}} \ln \left(\frac{p}{p_{\mathrm{s}}}\right)
$$

the approximation being justified by the small values of the vapor pressures compared to osmotic pressures (see SI, section 1).

Equation 15 indicates that in response to the local equilibration with the vapor when $p>p_{\mathrm{s}}$, the outer solution film gets diluted so that its osmotic pressure reaches a value below $\Pi_{\text {in }}$. This dilution is achieved by condensation of the vapor (Figure 2c, top). The osmotic pressure difference between the outside and inside of the sample described by Equation 15 results in a solvent flux from the outer film to the vein, driven by osmosis. This osmotic flux occurs even if the nanopores are not fully impermeable to the solute 21,22 (see below), and results in filling of the vein (bubble shrinkage). Symmetrically, for $p<p_{\mathrm{s}}$, the outer solution is concentrated instead of diluted (Figure 2c, bottom), and the osmotically-driven flux of solvent now results in emptying of the inner vein.

We note that the gradients of solute concentration existing in the situations depicted in Figure 2c also induce diffusion of the solute (red arrows). In the filling situation $\left(p>p_{\mathrm{s}}\right.$, Figure 2c top), the solute diffuses from the inside to the outside of the sample, tending to concentrate the solution in the external film. In order to maintain local thermodynamic equilibrium (Equation 14), condensation of the vapor into the film must occur to maintain the solute concentration (and thus the osmotic pressure) inside of the film constant, resulting in growth of the film (black arrows). The situation is reversed during emptying $\left(p<p_{\mathrm{s}}\right.$, Figure 2c bottom), where diffusion of solute back into the sample results in evaporation and film shrinkage.

In this regime with a film of solution covering the surface of the sample, it is thus possible 
to switch reversibly between the filling (Figure 2c, top) and the emptying (Figure 2c, bottom) situations by alternating the imposed vapor pressure between $p>p_{\mathrm{s}}$ (filling) and $p<p_{\mathrm{s}}$. As we explain below, the transition between filling and emptying is actually slightly shifted from $p_{\mathrm{s}}$ to $p_{0}<p_{\mathrm{s}}$ due to capillary effects.

Indeed, in addition to the osmotic driving forces, an additional, small capillary driving force associated with curvature of the liquid-vapor interface inside of the vein has to be taken into account. The pressure of the solution in the vein, $P_{\text {in }}$, is lower than the vapor pressure in the bubble, $p_{\mathrm{s}}$, i.e. $P_{\mathrm{in}}=p_{\mathrm{s}}+\Delta P_{\mathrm{c}}$ with

$$
\Delta P_{\mathrm{c}}=-2 \gamma \cos \theta\left(\frac{1}{W}+\frac{1}{H_{\mathrm{v}}}\right)<0 .
$$

for a vein with a rectangular cross-section $W \times H_{\mathrm{v}}$, and with $\theta$ the contact angle of the meniscus on the solid that we assume equal to zero here (hydrophilic walls). The pressure difference with the outside film at pressure $P_{\text {ext }}=p$ is thus

$$
\Delta P=P_{\text {ext }}-P_{\text {in }}=p-p_{\mathrm{s}}-\Delta P_{\mathrm{c}} \simeq-\Delta P_{\mathrm{c}}>0 .
$$

This driving force always favors the filling of the vein.

Filling and emptying of the veins thus occur under two types of driving forces: osmotic $(\Delta \Pi$, from Equation 15) and hydrostatic ( $\Delta P$, from Equation 17). We assume that transport limitations are within the nanopores. As a result, gradients are established in the nanoporous layer between the micro-veins and the sample edge, and the steady-state flow rate of the fluid, $Q\left[\mathrm{~m}^{3} / \mathrm{s}\right]$, is proportional to the quantities $\Delta P$ and $\Delta \Pi$ with a relation of the form ${ }^{22}$

$$
Q=\lambda \frac{k}{\eta}(\Delta P-\sigma \Delta \Pi)
$$

where we defined $Q$ as the filling flow rate (from the sample edge to the veins, positive when the veins are filling), $k\left[\mathrm{~m}^{2} / \mathrm{s}\right]$ is the permeability of the porous layer, $\eta[\mathrm{Pa}]$ is the fluid 
viscosity, $\lambda[\mathrm{m}]$ is a geometrical factor, ${ }^{32}$ and $\sigma \in[0-1]$ is a dimensionless reflection coefficient that accounts for the fact that the solute is not fully excluded from the pores, resulting in a partial osmotic effect ( $\sigma=1$ for a fully excluding membrane, $\sigma=0$ for a fully permeable membrane). ${ }^{21,22}$ The value of $\sigma$ thus depends on the interaction between the solute and the pore walls. For purely steric interactions, assuming a spherical solute of radius $r_{\mathrm{s}}$ and a pore of radius $r_{\mathrm{p}}$ (Figure 1a, inset), $\sigma$ takes the simple form ${ }^{21,22}$

$$
\sigma=\left[1-\left(1-\frac{r_{\mathrm{s}}}{r_{\mathrm{p}}}\right)^{2}\right]^{2}
$$

For a $r_{\mathrm{p}} \simeq 1 \mathrm{~nm}$ pore and a solute with $r_{\mathrm{s}}=0.17 \mathrm{~nm}$ like urea (see Methods), we expect $\sigma \simeq 0.1$ from Equation 19. We also note that using Equations 14, 15, 17, and neglecting the vapor pressures, Equation 18 can be rewritten

$$
Q=\lambda \frac{k}{\eta} \sigma\left(\Psi(p)-\Psi_{0}\right)
$$

where we have defined

$$
\Psi_{0}=\frac{\Delta P_{\mathrm{c}}}{\sigma}-\Pi_{\text {in }}
$$

the vapor water potential for which neither filling nor emptying occur $(Q=0)$. The corresponding vapor pressure, $p_{0}$, can be calculated from Equation 4, yielding the following simple expression (using Equation 7 to relate $\Pi_{\text {in }}$ to $p_{\mathrm{s}}$ and neglecting vapor pressures compared to osmotic and capillary pressures)

$$
p_{0}=p_{\mathrm{s}} \exp \left(\frac{v_{\mathrm{w}}^{\text {liq }} \Delta P_{\mathrm{c}}}{\sigma R T}\right)
$$

In our system, $\Delta P_{\mathrm{c}}=-0.064 \mathrm{MPa}$ and $\sigma=0.075$ so that $p_{0} / p_{\mathrm{s}} \simeq 0.994$. As a result, the transition between the filling and emptying regimes (Figure 2c) should occur very close to the equilibrium vapor pressure of the solution, $p_{\mathrm{s}}$. Larger deviations from $p_{\mathrm{s}}$ would occur in other systems with smaller vein dimensions (larger magnitude of the capillary pressure $\Delta P_{\mathrm{c}}$ ) 
or larger nanopores that would exclude the solute less (smaller value of $\sigma$ ). In general, inkbottle systems with a less extreme separation of lengthscales between the throats (nanopores) and the voids (veins) would lead to larger deviations of $p_{0}$ from $p_{\mathrm{s}}$ in Equation 22 .

\section{Osmotic stress transients}

The reversible filling and emptying by osmosis described in the previous section and Figure 2c are only dynamic, transient situations. Emptying ends when the bubble has expanded to the full volume of the vein, at which point filling can be triggered again by switching the vapor pressure to a value above $p_{\mathrm{s}}$. However, if filling is brought to completion, i.e. the bubble disappears, a new situation appears, where the vein is full with solution (Figure 2d, top). At this stage, if the pressure is brought back to $p<p_{\mathrm{s}}$, the situation does not reverse to the osmotic emptying case of Figure 2c (bottom), because there is no more bubble available for expansion to empty the micro-vein; instead, the situation is as described in Figure 2d, bottom, with a vein full of solution, and a receding film of solution at the surface of the sample. Obviously, this situation is only transient until the film completely disappears, at which point the equilibrium depicted in Figure 2a (center) and governed by Kelvin equation is established again. As we explain below, the situations in Figure 2d are associated with osmotic stresses (positive for $p>p_{\mathrm{s}}$ and negative for $p<p_{\mathrm{s}}$ ) that develop inside of the sample.

The situations of Figure 2d, similarly to those of Figure 2c described in the previous section, have a film of solution on the outside of the sample equilibrated with vapor. Consequently, the osmotic pressure imbalance between the film and the inside of the sample is still governed by Equation 15, with $\Delta \Pi=\Pi_{\text {ext }}-\Pi_{\text {in }}<0$ for $p>p_{\mathrm{s}}$ (Figure 2d, top) and $\Delta \Pi>0$ for $p<p_{\mathrm{s}}$ (Figure $2 \mathrm{~d}$, bottom). Since the vein remains full in both situations, there is no bulk solvent flow, and the osmotic driving force must be counter-balanced by a pressure difference, $\Delta P=P_{\text {ext }}-P_{\text {in }}$ of magnitude $\Delta P=\sigma \Delta \Pi$, according to Equation 18 
with $Q=0$. Using Equation 15 and neglecting the vapor pressures,

$$
P_{\text {in }}=\sigma \frac{R T}{v_{\mathrm{w}}^{\operatorname{liq}}} \ln \left(\frac{p}{p_{\mathrm{s}}}\right) .
$$

Equation 23 predicts that the system experiences an osmotic overpressure, $P_{\text {in }}>0$ when $p>p_{\mathrm{s}}$ (Figure 2d, top), and an osmotic negative pressure $P_{\text {in }}<0$ when $p<p_{\mathrm{s}}$ (Figure $2 \mathrm{~d}$, bottom). As in the previous section, as long as the outside film of solution exists, one can switch reversibly from one situation to the other at any time by changing the vapor pressure with respect to $p_{\mathrm{s}}$. We note from Equation 23 that $P_{\text {in }}$ only differs from the equilibrium pressure, $P$ predicted by Kelvin equilibrium (Figure 2a center) by a corrective factor $\sigma \leq 1$. Indeed, from Equation 8 (neglecting vapor pressures), the Kelvin stress is $P=R T \ln \left(p / p_{\mathrm{s}}\right)$. As a result, the stresses induced by steady-state osmosis are smaller than those implied by Kelvin equilibrium, except in the case of a perfect semi-permeable porous medium $(\sigma=1)$ for which they would be equal. From Equation 19, the osmotic stresses vanish quickly when the pore radius gets larger $\left(\sigma \simeq 4\left(r_{\mathrm{s}} / r_{\mathrm{p}}\right)^{2}\right.$ when $\left.r_{\mathrm{p}} \gg r_{\mathrm{s}}\right)$.

\section{Results and Discussion}

\section{Thermodynamics: evaporation and condensation isotherms}

We first recorded the state of the liquid in the veins when the sample was equilibrated with a subsaturated vapor (pressure, $p$ ). This observation was possible because of the clear optical difference between empty veins and veins full with liquid (Figure 1d). We performed these experiments both with pure water and with solution in the sample.

For the evaporation branch (referred to as emptying branch in the following), we started with an initially saturated sample, i.e. with both nanopores and micro-veins full with pure liquid water or aqueous solution. We then imposed a steady value of vapor pressure with $p / p_{\text {sat }}<1$ and counted the number of full and empty cavities after 6 hours (Fig. 3a, down 

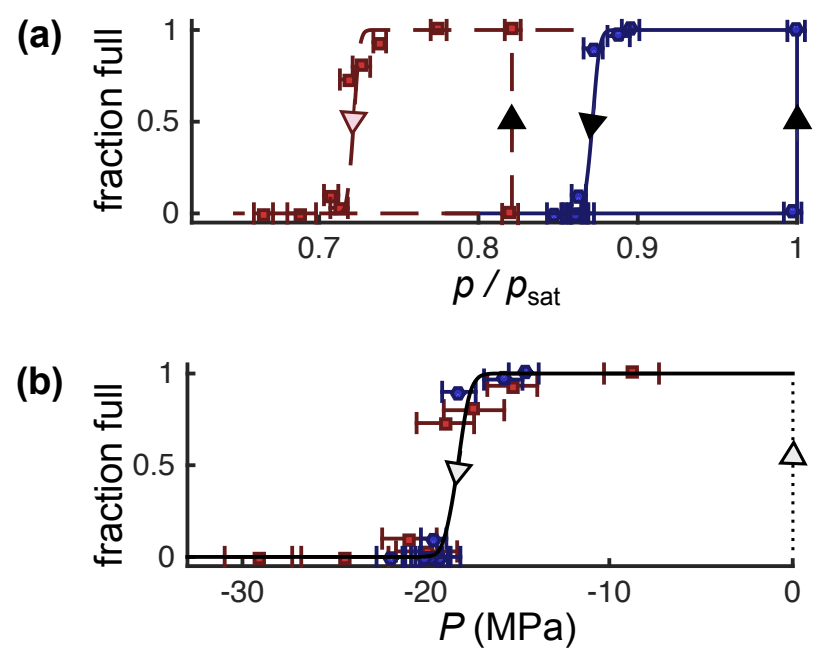

Figure 3: Thermodynamics: filling state of the veins with urea solution (red squares) and with pure water (blue circles). (a) Isotherms reporting the fraction of micro-veins filled as a function of vapor pressure. (b) Emptying branches re-plotted as a function of the calculated Kelvin pressure (Equations 9-10). Continuous and dashed lines are fits from classical nucleation theory for the emptying branches (SI, section 3). Similar results with lithium chloride solutions at different concentratoins are presented in the SI (section 5).

arrows). We repeated 6-hour steps of decreasing vapor pressures until the micro-veins in the sample were all empty.

For the condensation (filling) branch (Fig. 3a, up arrows), we started from a sample with empty veins and increased the vapor pressure (humidity) until visible filling occurred in the veins. For the sample filled with pure water, filling only occurred at saturation $\left(p / p_{\text {sat }}=1\right)$, while in the case of a solution-filled sample, filling occurred in a narrow range of vapor pressure around a value $p_{0}$ far from vapor saturation $\left(p_{\text {sat }}\right)$ but close to the equilibrium vapor pressure of the solution, $p_{\mathrm{s}}\left(p_{0} / p_{\text {sat }}=0.821\right.$ compared to $p_{\mathrm{s}} / p_{\text {sat }}=0.828$, see Methods and SI).

Both cases of pure water and urea solution displayed a qualitatively similar response, with a large hysteresis (Fig. 3a). The hysteresis cycle was shifted towards lower vapor pressures in the case of the solution, and its width was smaller than in the case of pure water.

The fact that filling occurred close to equilibrium for both water $\left(p=p_{\text {sat }}\right)$ and solution $\left(p=p_{\mathrm{s}}\right)$ in the micrometer-sized veins contrasts with the behavior of nanopores for 
which filling can occur far from equilibrium by capillary condensation. ${ }^{33}$ Recent estimates in our laboratory ${ }^{16,23}$ have shown that capillary condensation in the porous silicon nanopores constituting the nanoscale membrane in our samples occurs for $p / p_{\text {sat }} \simeq 0.6$, far below the vapor pressures investigated here. This observation suggests that the filling and emptying processes reported here in the micro-veins took place while the nanopores separating the veins from the vapor phase were always full with liquid. Although this fact can seem surprising at first sight, it is fully compatible with the model developed in the Theory section: emptying occurs far from equilibrium via the nucleation of vapor bubbles (see Theory, section Cavitation, desorption, and poroelastic emptying and Figure 2b) and thus does not require a vapor path to form between the external vapor and the vein. Filling, on the other hand, occurs close to equilibrium due to condensation of the vapor at the sample edge and the resulting filling flow driven by a combination of osmosis and capillarity (see Theory, section Filling-emptying by osmosis and capillarity and Figure 2c). Empyting by nucleation results from the development of negative pressure $(P<0)$ in the liquid for $p<p_{\mathrm{s}}$ (Equation 8 ), a metastable state that relaxes by cavitation. We expect cavitation to occur when the negative pressure becomes too large in magnitude, in a narrow range around $P_{\text {cav }}$ that we expect to be in the vicinity of $-20 \mathrm{MPa}$, as we have shown previously. ${ }^{16}$

We estimated the liquid pressure $P$ in the veins on the evaporation branch from the modified Kelvin relation (Equations 8-9), neglecting vapor pressures (Equation 10, i.e. $P=$ $\Psi(p)+\Pi_{\text {in }}$ with $\Psi(p)$ the vapor water potential as defined in Equation 4 and $\Pi_{\text {in }}$ the osmotic pressure of the solution filling the micro-veins and the nanopores). From the desorption branch in Figure 3a and Equation 10, we calculated the value of $P$ using the vapor pressure value $p$ of every data point, both for the urea solution $\left(\Pi_{\text {in }}=25 \mathrm{MPa}\right)$ and for pure water $\left(\Pi_{\mathrm{in}}=0\right)$, resulting in the data in Figure $3 \mathrm{~b}$. The data collapses onto a master curve, showing a sharp transition at a pressure $P_{\text {cav }} \simeq-18 \mathrm{MPa}$. The continuous lines in Figures 3a-b are fits to classical nucleation theory (see SI, section 3). We also obtained similar collapse and cavitation pressures with lithium chloride solutions of various concentrations (see SI, Figure 
S1a-b). These observations suggest that Equation 10 adequately describes the equilibrium stresses induced in a confined solution by liquid-vapor coexistence. As we will show below, these large tensile stresses implied by Kelvin equation also manifest themselves in dynamic situations, but can also be replaced in some regimes by transient stresses of lower magnitude dominated by osmotic processes.

\section{Dynamics: filling and emptying flows}

We investigated the dynamics of filling and emptying in our extreme ink-bottle porous systems in two distinct situations: complete filling and emptying refer to cases where all the micro-veins begin empty (resp. full) and end up full (resp. empty) under the action of a constant imposed vapor pressure, while partial cycles refer to cases where we prevent complete filling by oscillating the vapor pressure to produce a succession of filling and emptying situations.

\section{Complete emptying and filling}

We recorded the dynamics of filling and emptying of the extreme ink-bottle pores by measuring the advancement or retraction of liquid in the veins when subjected to constant conditions of vapor pressure. For filling, we started from a sample with empty veins (and filled nanopores) equilibrated with a vapor at $p<p_{\mathrm{s}}$, and then imposed a steady $p>p_{\mathrm{s}}$ after $t=0$. Inversely, for emptying, we started with full veins and imposed $p<p_{\mathrm{s}}$ at $t=0$. Experiments were conducted with both pure water and solution, and the rates of filling and emptying were obtained by image analysis by extracting the bubble length as a function of time in every vein of the sample (Figure 1d).

Typical results are shown in Figure 4 for filling and in Figure 5 for emptying. Filling and emptying displayed qualitative differences: while filling was a continuous and collective

process with all veins filling simultaneously (Figure 4), emptying occurred by discrete events well separated in time (Figure 5). Individual emptying events were extremely fast and 

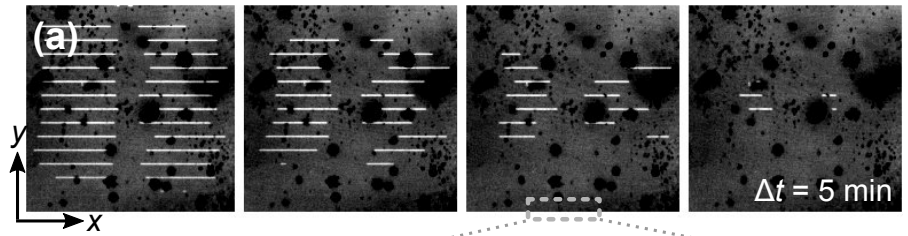

(b)

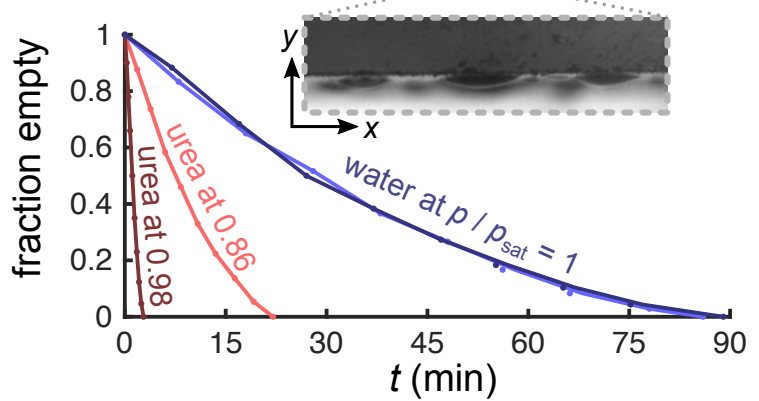

Figure 4: Dynamics of filling. (a) Typical image sequence (sample with urea solution with $p_{\mathrm{s}} / p_{\text {sat }}=$ 0.83 , filling in conditions with $p / p_{\text {sat }}=0.86$ of water vapor), with a time interval, $\Delta t=5$ minutes. Contrast has been increased to enhance the vein visibility. The dark spots visible on the sample were due to defects on the outer surface of the glass and did not impact the dynamics. The inset is a zoom showing the formation of bulk liquid in the form of droplets at the sample edge, where the nanoporous membrane was exposed to the vapor. (b) Comparison between the dynamics of filling with pure water in saturated vapor $\left(p / p_{\text {sat }}=1\right.$, blue curves, two experiments in the same conditions are shown) and the dynamics of filling in subsaturated water vapor $\left(p / p_{\text {sat }}<1\right)$ when the sample was filled with a urea solution (red curves). In both urea cases displayed, the urea solution filling the sample was the same $\left(p_{\mathrm{s}} / p_{\text {sat }}=0.83\right)$, but the imposed vapor pressure was different $\left(p / p_{\text {sat }}=0.98\right.$ and $p / p_{\text {sat }}=0.86$, indicated as urea at 0.98 and urea at 0.86 in the Figure, respectively). 

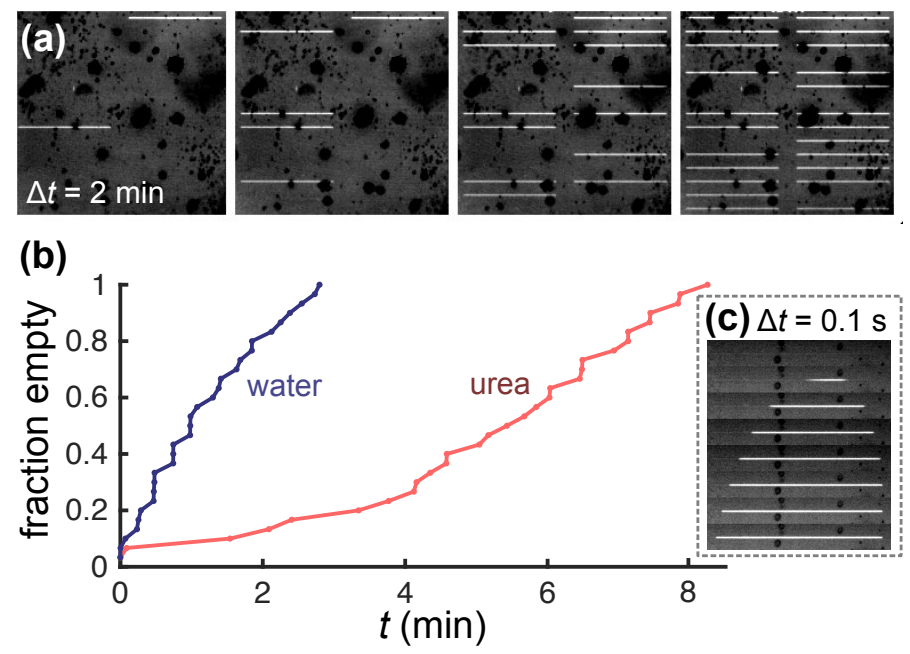

Figure 5: Dynamics of emptying. (a) Typical image sequence (sample with urea solution, emptying in $p / p_{\text {sat }}=0.60$ water vapor), with a time interval, $\Delta t=2$ minutes. Contrast has been increased to enhance the vein visibility. (b) Dynamics with urea solution (red curve) and water (blue curve), at $p / p_{\text {sat }}=0.60$ and $p / p_{\text {sat }}=0.85$, respectively. (c) Image sequence of a single micro-vein emptying after cavitation in water with a time interval, $\Delta t=0.1 \mathrm{~s}$ (see SI, section 6 for a detailed analysis of these individual emptying events). Cavitation occurred between the top-most frame and the frame just below.

unsteady (non-constant bubble growth rate), with bubble expansion times of $\simeq 1$ second (see Figure 5c and SI, section 6 for a detailed analysis of the dynamics of individual bubble expansion). In accordance with the equilibrium results described above (Figure 3), filling with pure water only occurred at $p=p_{\text {sat }}$ while filling with solution occurred for any situation where the imposed vapor pressure was higher than $p_{0} \simeq p_{\mathrm{s}}$, with faster filling rates for increasing vapor pressures. Also, no emptying occurred if the vapor pressure was not lowered sufficiently away from $p_{\mathrm{s}}$, reflecting the thermodynamic hysteresis of Figure 3.

Comparing filling and emptying with pure water, it is clear that the dynamics displayed a large asymmetry, with a slow filling (Figure 4b in blue) and fast emptying (Figure 5b, in blue). At first, the difference does not seem as pronounced in the case of the urea solution, since the total time to fill the sample at high vapor pressure was typically comparable or faster than emptying (Figures 4b-5b, red curves). However, as noted above, individual emptying events showed bubble growth rates several orders of magnitude faster than bubble 
collapse rates during filling (Figure $5 \mathrm{c}$ and SI, section 6).

\section{Partial emptying and filling cycles}

(a)

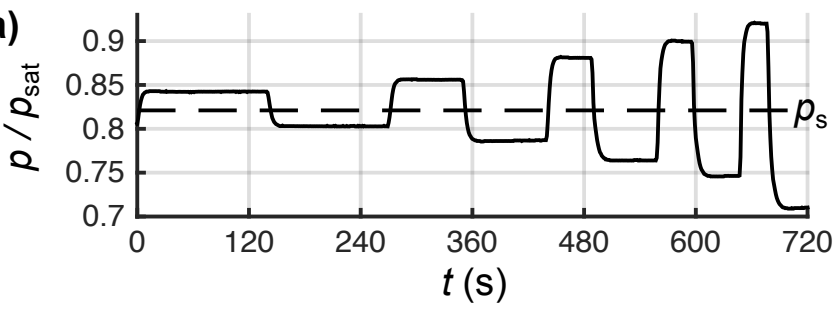

(b)
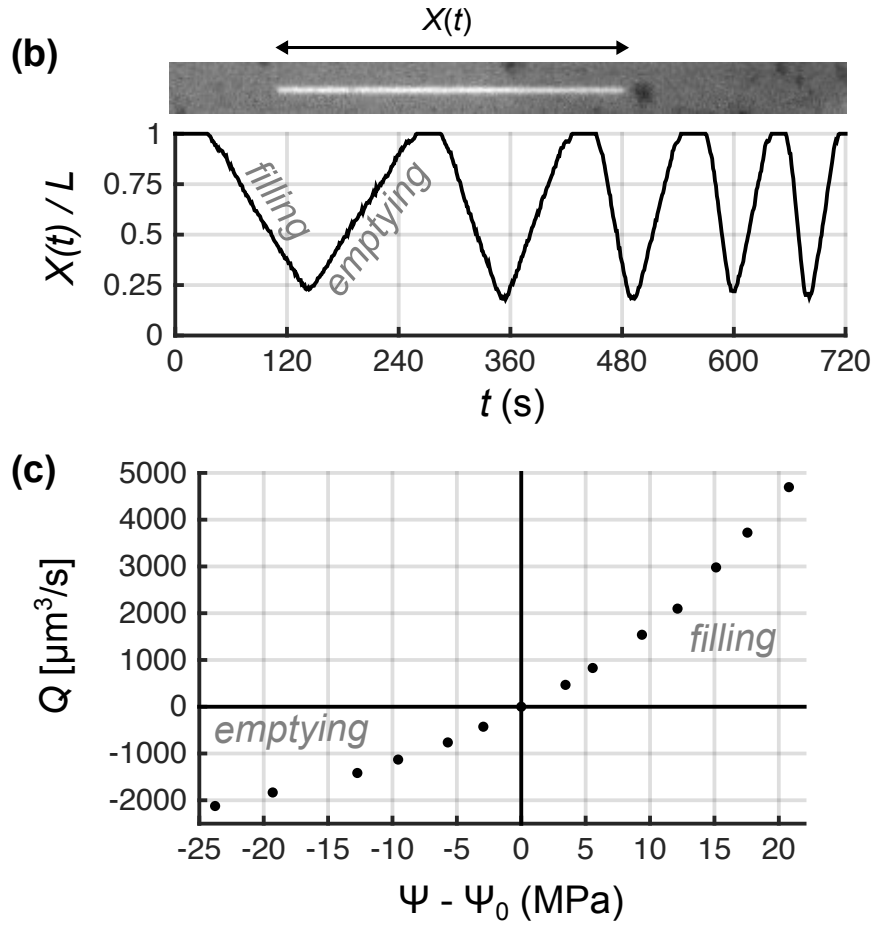

Figure 6: Dynamics of partial filling and emptying in a single micro-vein, achieved with oscillating external vapor pressure to prevent bubble collapse. Sample was filled with urea solution $\left(p_{\mathrm{s}} / p_{\text {sat }}=\right.$ 0.83). (a) Imposed vapor pressure variations. The dashed horizontal line corresponds to the solution equilibrium vapor pressure, $p_{\mathrm{s}}$. (b) Filling and emptying response, measured as a function of bubble length, $X(t)$, normalized by the micro-vein length, $L$. (c) Filling rate, $Q$, as a function of driving force. $\Psi_{0}$ is the vapor water potential at which neither filling nor emptying occurs, as measured experimentally.

In order to compare emptying and filling in a more symmetrical situation, we also ran experiments where we prevented the generation of strongly metastable states and their relaxation through cavitation by using a micro-vein with a pre-existing bubble. To do so, we 
first did a filling experiment as in Figure 4 with solution in the sample and $p>p_{0}$ until only one micro-vein still contained a bubble. Before this last bubble collapsed, we lowered the vapor pressure to a value below $p_{0}$ but not low enough to induce cavitation. As a result, the bubble started expanding again due to the induced emptying of the vein, without bubbles forming in other veins.

We then made this bubble undergo cycles of filling and emptying, making sure that the bubble never collapsed completely (Figure 6a-b). The results show a clear, steady-state response to both emptying and filling; this steady-state behavior contrasts dynamics due to cavitation that was largely unsteady, both globally (Figure 5a-b) and for single events (Figure 5c and SI, section 6). We extracted the flow rates from linear fitting of the responses as in Figure $6 \mathrm{~b}$ and the geometry of the veins, yielding the results reported in Figure 6c.

\section{General discussion}

It emerges from the results above that the comparison between filling and emptying is not trivial, and the qualitative difference in dynamics (e.g. continuous, collective filling vs. intermittent emptying mediated by fast, discrete events) indicates different physics at play.

\section{Kelvin equilibrium and filling-emptying isotherms}

We have shown from the results displayed in Figure 3 that isotherms of filling-emptying in the micro-veins of our sample were consistent with Kelvin expression for pure water (Equations 3-4 or Equations 8-9 with $\Pi=0$ and $p_{\mathrm{s}}=p_{\text {sat }}$ ) and the modified Kelvin expression (Equations 8-9) for a solution, yielding the following scenario.

Following the emptying (evaporation) branch, as the vapor pressure is lowered below $p_{\text {sat }}$ for pure water or $p_{\mathrm{s}}$ for the solution, the curvature of the liquid-vapor interface in the nanopores (Figure 2a, center) results in a large negative pressure (tension) in the liquid; this tension gets larger in magnitude for lower vapor pressures. At some point the stress is so large that bubbles spontaneously nucleate in the liquid in the micro-veins, triggering an 
emptying of the microcavities by cavitation and subsequent bubble expansion (Figure $2 \mathrm{~b}$ ). Using the Kelvin expressions, the pressure for cavitation was found to be $\simeq-18 \mathrm{MPa}$ for both water and urea solution (and lithium chloride solutions, see SI section 5), suggesting a weak effect of the presence of solute on the cavitation pressure. As discussed in the Theory section, it is unclear what physical mechanisms explain cavitation pressures around -20 MPa for water, but this value seem to be found in a variety of experiments, including some earlier studies from our group on similar silicon/glass extreme ink-bottle systems. ${ }^{16}$ Regardless of the cavitation mechanism, nucleation rates are expected to depend mostly on the pressure, $P$, in the liquid, ${ }^{28}$ so that emptying branches of isotherms originating from cavitation should be universal when plotted as a function of the calculated Kelvin pressure, $P$ in Equations 3 and 8-9, as we have demonstrated in Figures 3a-b and S1a-b.

On the filling (condensation) branch, we have shown filling at a vapor pressure $p_{0}$ very close to saturation ( $p_{\text {sat }}$ for water, $p_{\mathrm{s}}$ for solution). From Equations 3 and 8, this situation corresponds to $P \simeq 0$ (neglecting vapor pressures) so that filling isotherms also collapse when plotting them in terms of the Kelvin pressure, $P$ (Equations 3 and 8-9), in the form of a step jump from empty to full at $P=0$ (Figure $3 \mathrm{~b}$ ). We note that filling occurring at $p_{0} \simeq p_{s}$ $(P \simeq 0)$ is characteristic of the extreme ink-bottle geometry, due to the weak capillary pressure in the micro-veins. For regular ink-bottles without extreme size separation, filling should occur further away from saturation, $\left(p_{0}<p_{\mathrm{s}}\right.$ and $\left.P<0\right)$, as described by Equation 22. However, we expect that the collapse of isotherms when plotted in terms of Kelvin pressure $P$ should still exist.

\section{Cavitation and poroelastic emptying: unsteady dynamics}

The fast emptying dynamics after cavitation as in Figure 5c also provides a way to check the magnitude of the negative pressures generated prior to cavitation in the liquid. The excellent agreement between bubble expansion times measured experimentally (Figures 5c and S2) and our model (Equation 13 and SI section 6) indeed confirms that emptying flows 
are driven by $\simeq-18 \mathrm{MPa}$ driving forces that relax through a poroelastic process.

This poroelastic relaxation is also responsible for the intermittent dynamics observed experimentally (Figure 5b): as we have shown recently, ${ }^{16}$ this complex dynamics is typical of extreme ink-bottle porous media and comes from the large spatio-temporal fluctuations in pressure associated with the coupling between nucleation kinetics in the metastable liquid and poroelastic relaxations in the porous medium. We refer the reader to our previous work for a detailed analysis of these effects. ${ }^{16}$

\section{Steady-state filling: Kelvin vs capillarity vs osmosis}

In contrast to the dynamics of emptying by cavitation, the continuous filling dynamics (Figures 4 and 6) suggests a response to a quasi-steady driving force. Here, we examine the origin of this driving force and show that it is not governed by Kelvin equation, but is dominated by an osmotic process associated with the formation of a condensation film on the outside surface of the medium, with a weak contribution of the capillary pressure of the liquid-vapor interface in the micro-veins.

The dynamics of filling (Figures 4 and 6 ) for $p>p_{\mathrm{s}}$ showed a clear decrease of the filling time when $p$ is increased, demonstrating a vapor-pressure-dependent driving force for filling. At first sight, this response seems consistent with vapor pressure-dependent stresses implied by Kelvin equation: Equation 10 predicts that for $p>p_{\mathrm{s}}$, large positive pressures should be generated at the sample edge for a solution. However with pure water, generating such positive pressures would require $p>p_{\text {sat }}$ (see Equation 3), i.e., a vapor supersaturated with respect to bulk water condensation, a case that we do not consider here. As a result, the only driving force for filling with pure water is the negative capillary pressure $\Delta P_{\mathrm{c}}$ associated with the curvature of the liquid-vapor menisci inside of the microscale veins (Figure 2c). Following this reasoning, the rates of filling between pure water $\left(Q_{\mathrm{w}}\right)$ and solution $\left(Q_{\text {sol }}\right)$ should scale as $Q_{\mathrm{sol}} / Q_{\mathrm{w}} \simeq\left(\Psi+\Pi_{\mathrm{s}}\right) /\left(-\Delta P_{\mathrm{c}}\right)$. However, the ratio predicted by the latter expression is an order of magnitude higher than observed in the experiments (e.g., Figure 4); this discrepancy 
cannot be explained by the viscosity ratio between pure water and the solution. In other words, the driving force for filling is apparently not as large as predicted from the modified Kelvin equation.

The experimental observation of the formation of bulk droplets on the edge of the sample during filling at $p>p_{\mathrm{s}}$ (Figure $4 \mathrm{a}$, inset) suggests why the dynamics is different than predicted from Kelvin equation. Indeed, as we discussed in the Theory section, Equations 8-10 imply the formation of individual nano-menisci at each pore mouth supporting the large difference in pressure between liquid and vapor through Laplace equation. Bulk liquid present at the sample surface prevents the formation of these nano-menisci, as pore mouths are covered with liquid and not in contact with the external vapor. Kelvin equilibrium is thus not applicable in this situation. As explained in the Theory section, the origin of this external liquid is spontaneous condensation of the vapor at the surface when $p>p_{\mathrm{s}}$, because the vapor is then supersaturated with respect to the solution. Local equilibrium between that surface layer and the vapor imposes a solute concentration different than that inside of the sample, resulting in an osmotic imbalance. Filling dynamics is thus driven by osmosis, with a small extra capillary driving force from the meniscus inside of the micro-vein (Figure 2c). As illustrated by Equation 23, we expect that the stresses induced by osmosis are reduced by a factor $\sigma \simeq 0.1$ compared to Kelvin, thus explaining the Kelvin-based order-of-magnitude overestimation in the previous paragraph.

\section{Symmetrical emptying and filling responses in partial cycles}

From the discussion above, the stresses and associated dynamics should show a clear change of behavior between filling and emptying, with a strong, Kelvin-induced response for $p<p_{\mathrm{s}}$ (emptying), and $\mathrm{a} \sim 10$-fold reduced osmotic response for filling. This proposition is in apparent contradiction with the results of Figure 6c, which show a response that varies continuously across all vapor pressures when performing filling-emptying cycles.

As we show below, the solution of this contradiction lies in the existence, when imposing 
$p<p_{\mathrm{s}}$, of a transient phase dominated by osmosis, before the establishment of the Kelvin metastable equilibrium. As we discussed previously, imposing $p>p_{\mathrm{s}}$ results in the formation of a film of diluted solution on the sample surface (Figure 2c, top and Figure 4a, inset). This film grows in thickness over time, as solute is driven into the film by diffusion while further condensation from the vapor ensures that the outer solute concentration remains constant in the film to maintain local equilibrium with the vapor (Figure 2c, top). If the vapor pressure is now switched to $p<p_{\mathrm{s}}$, the situation is reversed in a symmetric way: the outer solution must increase in concentration to achieve equilibrium with the vapor, thus diffusion drives solute back into the pores, and evaporation into the vapor occurs to keep the film concentration constant (Figure 2c, bottom). With this picture of the processes, the filling and emptying processes are symmetrical, explaining the continuous behavior of the flow rates, governed by Equation 20.

Note that since in the osmotic emptying situation (Figure 2c, bottom) the outer solution layer shrinks over time, the film eventually disappears, and nano-menisci compatible with the pore-scale picture of the modified Kelvin equation (Figure 2a, center) can form again. There are thus two types of driving forces during emptying: when a transient film of solution is present at the sample surface, an osmotic driving force dominates, associated with a solute concentration larger on the outside of the sample than inside, while for longer times, a larger hydrostatic driving force develops, governed by Kelvin equation. The transition between these two regimes could explain the increase in emptying rate that can be observed in Figure $5 \mathrm{~b}$ for the urea solution after $t \simeq 4 \mathrm{~min}$.

\section{Magnitude of the osmotic effect: the reflection coefficient}

Since filling with pure water is driven by capillarity while filling with solution is driven by osmosis, comparison between the flow rates obtained in the case of pure water and in the case of a solution allows us to quantify the osmotic effect. Indeed, with pure water only the micro-vein capillary pressure, $\Delta P_{\mathrm{c}}=-0.064 \mathrm{MPa}$ (Equation 16) drives the filling 
flow. From Equation 18, $Q_{\mathrm{w}}=\lambda k\left(-\Delta P_{\mathrm{c}}\right) / \eta_{\mathrm{w}}$, with $\eta_{\mathrm{w}}$ the viscosity of pure water. In the presence of solute, the flow rate resulting from osmosis is given by Equation 20, i.e. $Q_{\text {sol }}=\lambda k \sigma\left(\Psi(p)-\Psi_{0}\right) / \eta_{\mathrm{s}}$, with $\eta_{\mathrm{s}}$ the viscosity of the solution. As a result, the ratio of filling flow rates

$$
\frac{Q_{\mathrm{sol}}}{Q_{\mathrm{w}}}=\sigma \frac{\eta_{\mathrm{w}}}{\eta_{\mathrm{s}}} \frac{\Psi(p)-\Psi_{0}}{\left(-\Delta P_{\mathrm{c}}\right)}
$$

provides access to the reflection coefficient $\sigma$, which quantifies the magnitude of the osmotic effect (see Theory).

Using the data in Fig. 6c from partial filling-emptying cycles, yields $\sigma=0.064$ if the data is linearized around $\Psi=\Psi_{0}$, with a range $\sigma=0.041-0.103$ across all data points due to the nonlinearity of the experimental response. Similarly, the complete filling dynamics data from Figure 4b can be used to measure the average filling flux as $Q=V_{\text {veins }} / \tau$ where $V_{\text {veins }}$ is the total combined volume of the veins, and $\tau$ is the total filling time. Comparing the filling rate for water (Figure $4 \mathrm{~b}$, blue) and for the urea solution at a variety of concentrations (Figure 4b red, and SI, Figure S1c) yields $\sigma=0.09 \pm 0.02$, using Equation 24. All these values (from partial cycles and from global filling dynamics) are comprised in the range

$$
\sigma=0.075 \pm 0.035
$$

a value that is in good agreement with theoretical estimates based on steric interactions between the solvent and the pore walls. Indeed, Equation 19 predicts $\sigma=0.065 \pm 0.032$ using the pore size estimate $1.4 \pm 0.4 \mathrm{~nm}$ obtained from $\mathrm{N}_{2}$ porosimetry. ${ }^{16,23} \mathrm{We}$ also checked the consistency of the above value of the reflection coefficient $\sigma$ by studying the competition of osmotic filling with inner gas pressure when performing experiments in air rather than in vacuum (see SI, section 7). We obtained similar, but slightly larger values of $\sigma$ from the analysis of lithium chloride experiments (see SI, section 5), suggesting that penetration of the solute into the pore is more hindered (larger $\sigma$ ) for lithium chloride than for urea.

It is unclear why the flux response as a function of the osmotic driving force as in Figure 6c 
is nonlinear. This observation could be the result of several effects, including the dependency of transport coefficients (viscosity, diffusivities) on the solute concentration, concentration polarization effects due to convection as the fluxes get larger in magnitude, and effects of concentrated diffusion. ${ }^{34}$

\section{General picture}

The data and discussion above yield the following picture for statics and dynamics of confined liquid-vapor equilibria in the presence of solutes, consistent with the diagrams in Figure 2.

First, considering thermodynamics along quasi-static isotherms, we have shown that the solutes impose a shift in the condensation-evaporation characteristics in the micro-veins that can be described by a modified Kelvin equation that includes the osmotic pressure of the solution (Equations 8-9). In the evaporation branch, strong metastability (negative pressure) develops until spontaneous cavitation induces emptying of the micro-veins, while on the condensation branch filling of the veins occurs close to equilibrium (saturation), driven by capillarity in the micro-veins. Saturation occurs at $p=p_{\text {sat }}$ for water, and $p=p_{\mathrm{s}}<p_{\text {sat }}$ for a solution.

Second, we have considered the dynamics of filling and emptying in out-of equilibrium situations. At vapor pressures low enough to induce cavitation, we observed emptying dynamics dominated by discrete nucleation events followed by fast, unsteady, poroelastic bubble expansion driven by the large Kelvin stresses. Filling, on the other hand, occurred at high vapor pressure. With pure water, filling only occurred by capillarity at $p=p_{\text {sat }}$, but solutions allow us to explore situations where the vapor is actually supersaturated with respect to the equilibrium vapor pressure of the solution, i.e. $p_{\mathrm{s}}<p<p_{\text {sat }}$. We have shown that the resulting condensation on the sample edge triggers a faster filling process dominated by osmosis, which is directly controlled by the degree of supersaturation, $p / p_{\mathrm{s}}$ of the vapor.

We have also explored filling-emptying cycles induced by oscillating the vapor pressure above and below $p_{\mathrm{s}}$ when a film of solution is present at the sample surface. With a partially 
empty micro-vein, the oscillation results in more symmetrical filling and emptying flows both driven by osmosis, as long as a bubble is maintained in the vein (Figure 2c). If the bubble collapses, potentially large, transient osmotic stresses are generated, with a sign that depends on the saturation of the vapor with respect to $p_{\mathrm{s}}$.

\section{Conclusion}

We have studied the thermodynamics and dynamics of condensation and evaporation from extreme ink-bottle porous structures (micro-veins combined with nanopores) filled with aqueous solutions. We have shown that emptying of the micro-veins upon evaporation occurred far from equilibrium due to the generation of metastability (negative pressure) in the liquid, relaxing by cavitation, i.e., the nucleation of vapor bubbles. Inversely, filling during condensation proceeded at near equilibrium conditions. This striking difference between evaporation and condensation resulted in large, steep hysteresis of the filling state of the medium as a function of vapor pressure.

We have demonstrated that the tensile stresses in the liquid were well described by a modified Kelvin equation that takes into account the osmotic pressure of the solution. These large tensile stresses played a direct role in the kinetics of cavitation (classical nucleation theory) and in the fast, poroelastic expansion dynamics of bubbles after nucleation. On the contrary, we showed that Kelvin equation failed to describe positive liquid pressures, due to the formation of bulk solution films on the outer edge of the sample; these films are incompatible with the presence of pore-scale nano-menisci. In this situation, the driving forces were due to partial osmosis (reflection coefficient, $\sigma<1$ ), resulting in limited stresses compared to Kelvin equation. In fact, we showed that this osmosis effect could also play a role in the negative-pressure regime, by replacing the Kelvin effect when the sample is temporarily covered with a layer of solution, resulting in a steady-state transient of lower stress and slower emptying fluxes compared to those induced by Kelvin stresses. The resulting relaxation 
dynamics under vapor pressure changes reflected the various driving forces, with symmetrical, slow osmotic filling-emptying dynamics at short timescales, and significantly faster emptying by cavitation at longer timescales due to the large Kelvin stresses.

We note that although we have only analyzed the effect of Kelvin and osmotic stresses from the bubble nucleation and dynamic flow response that they generated, these stresses (that can be both tensile and compressive depending on the imposed vapor pressure) should also impact the solid matrix and trigger reversible deformations or potential damage, depending on the mechanical characteristics of the material. This opens perspectives to extend existing studies of the impact of liquid-vapor equilibria on the mechanics of materials (e.g. granular cohesion ${ }^{4}$ or elastocapillarity ${ }^{35}$ ) to the case of solutions, with the new phenomena (osmosis, shifted Kelvin) elucidated here.

Globally, the results reported in this paper a) show the usefulness of the extreme inkbottle platforms for the study of basic processes in porous media, with convenient optical access and direct visualization of the filling state of the larger pores (veins), b) elucidate the interplay between liquid-vapor equilibria (Kelvin), capillarity and osmosis when the pores are filled with solution, providing a solid basis to model more complex porous media, c) can be applied to technologies using stresses induced by evaporation-condensation such as tensiometers, ${ }^{36}$ heat pipes ${ }^{37}$ and actuators. ${ }^{38}$

Some important aspects not considered in this paper deserve to be mentioned. First, by working in vacuum we have eliminated complications due to the presence of air. While we do not expect air to modify appreciably the thermodynamic equilibria investigated here, its presence could impact dynamics significantly. As illustrated by the complementary experiments described in the SI (section 7), air pressure adds an extra driving force, which depends on how fast air diffuses into the solution-filled matrix. Future work should elucidate the complex interplay between different transport mechanisms and driving forces. Second, we have focused on filling and emptying of the microstructure (veins) in situations where the nanopores stayed full with liquid. Going to vapor pressures low enough so that the 
nanoporous matrix itself starts drying brings new phenomena, such as desorption, solution concentration, and crystallization of the solute. We investigate this situation in an upcoming article.

We finally note that most of the results obtained here for extreme ink-bottles are applicable to more general pore architectures, in particular the modified Kelvin equation and the effects of osmosis on filling and emptying dynamics. The specificity of ink-bottles is that emptying occurs by bubble nucleation rather than the invasion of vapor from the edge.

\section{Acknowledgement}

The authors thank Glenn Swan for technical support. This work was supported by the National Science Foundation (IIP-1500261), the Air Force Office of Scientific Research (FA955015-1-0052), the U.S. Department of Agriculture (2015-67021-22844) and the Camille Dreyfus Teacher-Scholar Awards program, and was performed in part at the Cornell NanoScale Facility, a member of the National Nanotechnology Infrastructure Network (National Science Foundation; Grant No. ECCS-1542081).

\section{Supporting Information Available}

Supporting Information (SI) is available: derivation of thermodynamic relations in solutions, classical nucleation theory for cavitation, experimental measurement of solution activity and osmotic pressure, additional experimental results with lithium chloride, experimental results and modeling of poroelastic emptying, and an alternative measurement of osmotic effects using competition with air pressure. 


\section{References}

(1) Al-Muhtaseb, A.; McMinn, W.; Magee, T. Moisture sorption isotherm characteristics of food products: a review. Food Bioprod. Process 2002, 80, 118-128.

(2) Marcolli, C. Deposition nucleation viewed as homogeneous or immersion freezing in pores and cavities. Atmos. Chem. Phys. 2014, 14, 2071-2104.

(3) Stroock, A. D.; Pagay, V. V.; Zwieniecki, M. A.; Holbrook, N. M. The Physicochemical Hydrodynamics of Vascular Plants. Annu. Rev. Fluid Mech. 2014, 46, 615-642.

(4) Bocquet, L.; Charlaix, E.; Ciliberto, S.; Crassous, J. Moisture-induced ageing in granular media and the kinetics of capillary condensation. Nature 1998, 396, 735-737.

(5) Jensen, O. M.; Hansen, P. F. Autogenous deformation and RH-change in perspective. Cem. Concr. Res. 2001, 31, 1859-1865.

(6) Zwieniecki, M. A.; Holbrook, N. M. Confronting Maxwell's demon: biophysics of xylem embolism repair. Trends Plant Sci. 2009, 14, 530-534.

(7) Powers, T. Freezing effects in concrete. ACI Special Publication 1975, 47, 1-12.

(8) Valenza, J. J.; Scherer, G. W. A review of salt scaling: II. Mechanisms. Cem. Concr. Res. 2007, 37, 1022-1034.

(9) Neimark, A. V.; Ravikovitch, P. I.; Vishnyakov, A. Bridging scales from molecular simulations to classical thermodynamics: density functional theory of capillary condensation in nanopores. J. Phys.: Condens. Matter 2003, 15, 347-365.

(10) Factorovich, M. H.; Solveyra, E. G.; Molinero, V.; Scherlis, D. A. Sorption Isotherms of Water in Nanopores: Relationship Between Hydropohobicity, Adsorption Pressure, and Hysteresis. J. Phys. Chem. C 2014, 118, 16290-16300. 
(11) Ravikovitch, P. I.; Neimark, A. V. Experimental Confirmation of Different Mechanisms of Evaporation from Ink-Bottle Type Pores: Equilibrium, Pore Blocking, and Cavitation. Langmuir 2002, 18, 9830-9837.

(12) Wallacher, D.; Künzner, N.; Kovalev, D.; Knorr, N.; Knorr, K. Capillary Condensation in Linear Mesopores of Different Shape. Phys. Rev. Lett. 2004, 92, 195704.

(13) Mistura, G.; Pozzato, A.; Grenci, G.; Bruschi, L.; Tormen, M. Continuous adsorption in highly ordered porous matrices made by nanolithography. Nat. Commun. 2013, 4, 2966.

(14) Bruschi, L.; Mistura, G.; Liu, L.; Lee, W.; Gsele, U.; Coasne, B. Capillary condensation and evaporation in alumina nanopores with controlled modulations. Langmuir 2010, 26, 11894-11898.

(15) Grosman, A.; Ortega, C. Cavitation in metastable fluids confined to linear mesopores. Langmuir 2011, 27, 2364-2374.

(16) Vincent, O.; Sessoms, D. A.; Huber, E. J.; Guioth, J.; Stroock, A. D. Drying by Cavitation and Poroelastic Relaxations in Porous Media with Macroscopic Pores Connected by Nanoscale Throats. Phys. Rev. Lett. 2014, 113, 134501.

(17) Nelson, P. H. Pore-throat sizes in sandstones, tight sandstones, and shales. AAPG Bull. 2009, 93, 329-340.

(18) Parlange, J.-Y. Water movement in soils. Geophysical Surveys 1974, 1, 357-387.

(19) Cohan, L. H. Sorption hysteresis and the vapor pressure of concave surfaces. J. Am. Chem. Soc. 1938, 60, 433-435.

(20) Cole, M. W.; Saam, W. Excitation spectrum and thermodynamic properties of liquid films in cylindrical pores. Phys. Rev. Lett. 1974, 32, 985-988. 
(21) Anderson, J. L.; Malone, D. M. Mechanism of osmotic flow in porous membranes. Biophys. J. 1974, 14, 957-982.

(22) Deen, W. Hindered transport of large molecules in liquid-filled pores. AIChE J. 1987, 33, 1409-1425.

(23) Vincent, O.; Szenicer, A.; Stroock, A. D. Capillarity-driven flows at the continuum limit. Soft Matter 2016, 12, 6656-6661.

(24) Chen, I.-T.; Sessoms, D. A.; Sherman, Z. M.; Choi, E.; Vincent, O.; Stroock, A. D. Stability Limit of Water by Metastable Vapor-Liquid Equilibrium with Nanoporous Silicon Membranes. J. Phys. Chem. B 2016, 120(23), 5209-5222.

(25) Vincent, O.; Marguet, B.; Stroock, A. D. Imbibition triggered by capillary condensation in nanopores. Langmuir 2017, 33, 1655-1661.

(26) Kawahara, K.; Tanford, C. Viscosity and density of aqueous solutions of urea and guanidine hydrochloride. J. Biol. Chem. 1966, 241, 3228-3232.

(27) Gosting, L. J.; Akeley, D. F. A Study of the Diffusion of Urea in Water at 25C with the Gouy Interference Method. J. Am. Chem. Soc. 1952, 74, 2058-2060.

(28) Debenedetti, P. G. In Metastable Liquids: Concepts and Principles; Prausnitz, J. M., Brewer, L., Eds.; Princeton University Press, 1996.

(29) Caupin, F.; Herbert, E. Cavitation in water: a review. C. R. Phys. 2006, 7, 1000-1017.

(30) Caupin, F.; Stroock, A. D. The stability limit and other open questions on water at negative pressure. Liquid Polymorphism: Advances in Chemical Physics 2013, 152, 51-80.

(31) Vincent, O.; Marmottant, P.; Quinto-Su, P. A.; Ohl, C.-D. Birth and growth of cavitation bubbles within water under tension confined in a simple synthetic tree. Phys. Rev. Lett. 2012, 108, 184502. 
(32) Consider a porous medium filled with liquid, with a pressure difference $\Delta P[\mathrm{~Pa}]$ applied between two cross-sections of area $\mathcal{A}\left[\mathrm{m}^{2}\right]$ across a length $\ell[\mathrm{m}]$. According to Darcy's law, a steady-state flow rate $Q\left[\mathrm{~m}^{3} / \mathrm{s}\right]$ develops because of the pressure gradient $\nabla P=$ $\Delta P / \ell$, resulting in $Q=\mathcal{A} \nabla P k / \eta$ where $k$ is the intrinsic permeability of the porous medium and $\eta$ the viscosity of the fluid. This latter expression can be rewritten as $Q=\lambda k \Delta P / \eta$ with $\lambda=\mathcal{A} / \ell[\mathrm{m}]$. This relationship should hold in more complex flow geometries, in which case $\lambda$ is an effective lengthscale. In our sample we expect $\mathcal{A} \sim$ $H_{\mathrm{p}} L$. Using $H_{\mathrm{p}}=15 \mu \mathrm{m}, L=2 \mathrm{~mm}$, and $\ell=300 \mu \mathrm{m}$, we thus estimate $\lambda \sim 100 \mu \mathrm{m}$.

(33) Charlaix, E.; Ciccotti, M. In Handbook of Nanophysics: Principles and methods.; Sattler, K., Ed.; CRC Press, 2010; Chapter Capillary condensation in confined media, pp $12.1-12.17$.

(34) Cussler, E. L. Diffusion: mass transfer in fluid systems, 3rd ed.; Cambridge University Press, 2009.

(35) Gor, G. Y.; Bertinetti, L.; Bernstein, N.; Hofmann, T.; Fratzl, P.; Huber, P. Elastic response of mesoporous silicon to capillary pressures in the pores. Appl. Phys. Lett. 2015, 106, 261901.

(36) Pagay, V.; Santiago, M.; Sessoms, D. A.; Huber, E. J.; Vincent, O.; Pharkya, A.; Corso, T. N.; Lakso, A. N.; Stroock, A. D. A microtensiometer capable of measuring water potentials below -10 MPa. Lab Chip 2014, 14, 2806-2817.

(37) Chen, I.-T.; Pharkya, A.; Stroock, A. D. Analysis of superheated loop heat pipes exploiting nanoporous wick membranes. AIChE J. 2014, 60, 762-777.

(38) Ma, M.; Guo, L.; Anderson, D. G.; Langer, R. Bio-inspired polymer composite actuator and generator driven by water gradients. Science 2013, 339, 186-189. 


\section{Graphical TOC Entry}

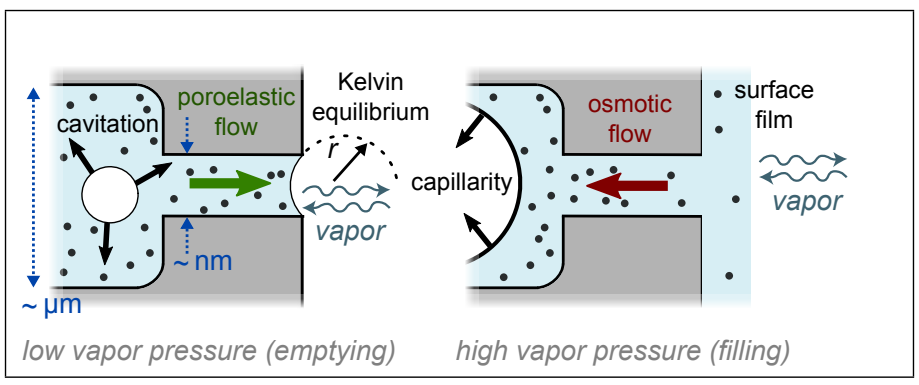

